\title{
Non-Market Wealth, Background Risk and Portfolio Choice ${ }^{1}$
}

Günter Franke ${ }^{2}$, Harris Schlesinger ${ }^{3}$, Richard C. Stapleton, ${ }^{4}$

March 1, 2007

${ }^{1}$ We thank Jens Jackwerth, Jack Meyer and Claus Munk for helpful comments. We also benefitted from comments by seminar partcipants at the Universities of Pennsylvania, Melbourne, Lancaster, Manchester and Ulm as well as particpants at the meeting of the European Group of Risk and Insurance Economists in Marseille and the 10th Symposium on Finance, Banking and Insurance in Karlsruhe.

${ }^{2}$ Univerity of Konstanz, Germany

${ }^{3}$ University of Alabama, USA

${ }^{4}$ University of Manchester, UK. 


\section{Abstract \\ Non-Market Wealth, Background Risk and Portfolio Choice}

We examine the effects of non-portfolio risks on optimal portfolio choice. Examples of non-portfolio risks include, among others, uncertain labor income, uncertainty about the terminal value of fixed assets such as housing and uncertainty about future tax liabilities. In particular, while some of these risks are added to portfolio value and have been amply studied, others are multiplicative in nature and have received far less attention. Moreover, the combined effects of multiple risks lead to some seemingly paradoxical choice behavior. We rationalize such behavior and we show how non-portfolio risks might lead to seemingly U-shaped relative risk aversion for a representative investor, as found empirically by Ait-Sahilia and Lo (2000) and Jackwerth (2000).

JEL classification: G 11

Keywords: Portfolio choice, Derived relative risk aversion, Additive background risk, Multiplicative background risk 


\section{Introduction}

It is well known that non-portfolio assets play a non-trivial role in deciding upon an investment strategy. For example, labor income and its inherent risks can play a substantial role in portfolio choice. ${ }^{1}$ Or consider an employee saving for retirement, who is expecting to receive a large inheritance from her rich uncle sometime before she retires. A promised $\$ 100,000$ inheritance at some point in the future is no different than owning a zero-coupon bond today. That is of course as long as the promise is kept. But what if the uncle is not quite so rich; or what if the uncle does not die prior to his niece's retirement; or what if the uncle never really liked his niece anyway; or what if the uncle actually had twice as much money as everyone thought?

In general, the expected inheritance (or expected future salary) tends to make one's observed behavior seem less risk averse, since the individual buys fewer bonds for her investment portfolio. On the other hand, as shown by Eeckhoudt and Kimball (1992), Gollier and Pratt (1996) and by Viceira (2001), the riskiness of this future source of wealth can lead to observed behavior that seems more risk averse, ceteris paribus.

As we show below, the interaction of these two effects alone is enough to make an individual whose underlying preferences exhibit constant relative risk aversion (CRRA) behave in financial markets as if her risk aversion was U-shaped, exhibiting decreasing relative risk aversion (DRRA) at low wealth levels followed by increasing relative risk aversion (IRRA) at higher wealth levels. Such an observation is not innocuous. Indeed in a very well crafted empirical study, Ait-Sahalia and Lo (2000) back out the risk aversion of a representative agent in an equilibrium setting using market data and they show that observed relative risk aversion exhibits such U-shaped behavior. A similar result was found independently by Jackwerth (2000).

In addition to such non-portfolio types of assets, other types of outside risks might also affect portfolio choice. For example, an individual saving for a particular purpose will be concerned with the purchasing power of her future portfolio wealth. Someone saving for a future college education for her son,

\footnotetext{
${ }^{1}$ See, for example Bodie et al (1992), Viceira (2001), Campbell and Viceira (2002) and Coco et al (2005).
} 
for example, will be concerned with future tuition costs and other education related expenses. Although some inflation hedging may be possible, there will be some residual risk that is not. This is especially true for a long-horizon investor. Moreover, unlike the additive nature of non-portfolio assets, such risks are often multiplicative in nature: the purchasing power of the whole investment portfolio might be one percent higher than expected, or two percent lower.

One current direction of research looks towards the design of investment instruments for such types of purposes. For example, papers by Bodie (2003) and Merton (2003), as well as the book by Shiller (2003), suggest that future research along these lines needs to be a primary focus in finance. However, little has been done to examine the effects of such risks on simple portfolioinvestment strategies. For convenience, we label these risks, which affect portfolio behavior but are not attached to the riskiness of the market assets within a portfolio, as "background risks." In this paper we examine asset allocation strategies for investors who face such types of "background risks." We examine both additive and multiplicative types of background risks and demonstrate some peculiar effects that might occur when both types of risks exist concurrently.

We simplify the generic problem above by assuming that the background risks are all independent of each other as well as independent of the market risks associated with the stock portfolio. This is not done for the sake of realism. Surely real-world examples of such background risks might be correlated with other risks. However, such correlations lead to well-known cross hedging strategies, which are not a part of our focus here. Instead we are interested in the pure risk effects: How do such background risks, when viewed as "noise," affect individual portfolio choice?

In line with a long tradition in portfolio theory, starting with Merton (1969, 1971), we assume that the investor is constant relative risk averse and that the market portfolio follows a geometric random walk. Merton (1971) showed that such an investor follows a portfolio allocation strategy that would invest a constant proportion of wealth in stocks, in the absence of any background risks. In a dynamic setting, such an investor would always rebalance her portfolio back to this constant proportion, following any change in asset valuation. This is our benchmark case. Our purpose in this paper is to 
show how observed behavior differs from this benchmark in the presence of background risks. ${ }^{2}$

A good analogy might be as follows. Suppose that you can observe a representative investor on the trading floor, but you are not able to observe these background risks. What is it that you are likely to observe? Take the simplest case of a fixed non-market wealth that the investor will receive at the end of the trading period, say $\$ 100,000$. Since the $\$ 100,000$ is a perfect substitute for market-purchased bonds, the first likely observation is that the individual does not seem very risk averse, since she invests most if not all of her wealth in stocks. However, suppose that stock prices rise dramatically. Since the \$100,000 fixed non-market wealth has not changed, our CRRA investor needs to buy proportionately more bonds in order to rebalance her portfolio. Observing this behavior, our investor appears to become more risk averse as she gets wealthier. Thus, our likely observation is of someone with a lower level of risk aversion than the CRRA preferences would indicate, and of someone not with CRRA preferences at all, but rather with relative risk aversion that is increasing in wealth. ${ }^{3}$

If the inheritance is risky, we show how this added risk on its own leads to more cautious investment behavior, i.e. investing in a higher proportion of risk-free bonds. If we also add a multiplicative risk, such as uncertain future tax rates on investment gains, the overall effect can become quite unusual. For example, under CRRA, it follows trivially that an independent multiplicative risk on the terminal portfolio value, in the absence of any non-portfolio wealth, would have no effect on the proportion of stocks and bonds held in the optimal portfolio. However, this does not imply that the multiplicative risk does not affect the investor. The extra risk makes the investor worse off. It is just that there is no change in the optimal portfolio

\footnotetext{
${ }^{2}$ We use the assumption of CRRA with geometric Brownina motion due to their historic place in the finance literature. However, our main purpose is to demonstrate how any "base case" scenario can be altered by the existence of these "background risks."

${ }^{3}$ In a very thought-provoking paper, Meyer and Meyer (2005) look at empirical research about the level of risk aversion in CRRA models over the years and they explain much of the difference in the empirical estimates by the use of different measures of "total wealth" for an individual. If total wealth is only portfolio wealth, for example, the estimates are much different that when total wealth includes items such as pension wealth and/or the value of owned real estate. See also Coco (2005).
} 
of stocks and bonds. Moreover, as we illustrate in the paper, in the presence of risky non-portfolio assets, this multiplicative risk affects the investor's reaction to other background risks and, hence, her portfolio choice.

In the next section, we outline the basic portfolio model that we will use throughout. We set up a somewhat common stylized model that includes CRRA preferences as a base model. The main point we wish to make is that various background risks can affect the behavior that one observes in the financial marketplace. One can also start with other "base cases" with similar types of results. Also, we stress at the outset that we look only at observed behavior for one individual and not at any type of general equilibrium. The focus is on how background risks alter portfolio choice for a given set of asset prices.

The outline of this paper is as follows. In the following section, we set up the base model for portfolio choice absent any consideration of non-market wealth or background risks. In section 3, we show how the presence of nonmarket wealth affects portfolio decisions. Section 4 considers the inclusion of a multiplicative background risk, which would not alter portfolio decisions in the absence of non-market wealth, but does alter these decisions in the presence of non-market wealth. In section 5, we illustrate our results by considering a numerical simulation of investment behavior in a state-contingent claims framework. We then decompose this demand for contingent claims into dynamic stock/bond allocation decisions in section 6, prior to concluding the paper.

\section{Basic Portfolio Model}

In this section, we set up the base model that we use throughout the rest of the paper. Consider a risk-inverse investor who maximizes the expected utility of wealth over a fixed investment horizon. We consider a pure investment problem and ignore intermediate consumption. We assume that preferences are derived over the terminal value of wealth. For the sake of concreteness, we assume that these preferences exhibit constant relative risk aversion, with marginal utility of wealth $w$ given by $u^{\prime}(w)=w^{-\gamma}$, where 
$\gamma>0$ denotes the degree of relative risk aversion. ${ }^{4}$

Let $\widetilde{R}$ denote the gross return on the market. The optimal investment policy can be described by a demand function $x(R)$ derived from a static one-period model. Letting $\phi(R)$ denote the pricing kernel, i.e. the price per unit of probability for a contingent claim with a payout of 1 in "state" $R$, the investor's objective can be written as

$$
\max _{x(R)} E[u(x(\widetilde{R}))] \text {, s.t. } E[\phi(\widetilde{R}) x(\widetilde{R})]=x_{0},
$$

where $x_{0}$ denotes the investor's market wealth at date $t=0$.

The first-order conditions for optimizing (1) are the budget constraint together with the conditions

$$
u^{\prime}(x(R))=\lambda \phi(R), \forall R,
$$

where $\lambda$ is the Lagrange multiplier for (1). ${ }^{5}$ Differentiating (2) with respect to $R$ and then using (2) to replace $\lambda$ in the result, we obtain

$$
x^{\prime}(R)=\frac{\phi^{\prime}(R) / \phi(R)}{u^{\prime \prime}(x(R)) / u^{\prime}(x(R))} .
$$

Letting $A(x) \equiv-x u^{\prime \prime}(x) / u^{\prime}(x)$ denote the Arrow-Pratt measure of relative risk aversion, it follows from (3) that the optimal contingent claim $x(R)$ satisfies

$$
[A(x)]^{-1}=-\frac{x^{\prime}(R) \phi(R)}{x(R) \phi^{\prime}(R)}=-\frac{d \ln x(R)}{d \ln \phi(R)}
$$

Using (4), it follows that

$$
\frac{d \ln x}{d \ln R}=\left[-\frac{d \ln x}{d \ln \phi}\right] \cdot\left[-\frac{d \ln \phi}{d \ln R}\right]=[A(x)]^{-1} \cdot\left[-\frac{d \ln \phi}{d \ln R}\right] .
$$

\footnotetext{
${ }^{4}$ As is well known in this case, utility takes the form $u(w)=\ln w$ for $\gamma=1$. Otherwise, $u(w)=(1-\gamma)^{-1} w^{1-\gamma}$.

${ }^{5}$ The value $\lambda$ equals the marginal utility of the initial market wealth. Note that for a CRRA investor her marginal utility covers the whole set of positive numbers. Therefore, given the usual integrability conditions, the optimal solution is interior (see Back and Dybvig (1993)).
} 
The first term on the right-hand side above is a positive constant (equal to $1 / \gamma)$ due to our assumption of CRRA utility for total wealth. Given that we also assume a geometric Brownian motion for the evolution of $\widetilde{R}$, the second term (i.e. the elasticity of the pricing kernel) is also a positive constant. ${ }^{6}$ Then, $\ln x(R)$ is an increasing and linear function of $\ln R$. That is, the investor has a log-linear demand function for contingent claims. Moreover, this linear function is flatter, ceteris paribus, for a higher level of relative risk aversion.

\section{Derived Risk Aversion Given Non-market Wealth}

In this section we consider the effects of non-market wealth on the derived risk aversion of an agent. Both the expected level of non-market wealth available as well as its risk play important roles in the analysis.

Let $z$ denote the net value of non-stochastic non-market wealth available at the horizon date. A positive $z$ is equivalent to the payoff on a zero-coupon bond. As a result, there is less discretionary investment in bonds, making the investor seem "as if" she is less risk averse. Similarly, if the market portfolio return is higher than the risk-free return, the investor with CRRA preferences wishes to re-balance her portfolio, but this would require a disproportionately higher investment in bonds than CRRA would indicate, making the investor seem "as if" her preferences exhibit increasing relative risk aversion.

If $z$ is negative, the investor behaves "as if" she was endowed with a short position in bonds. This investor would purchase more bonds on her discretionary account than would be the case if $z$ were zero, her behavior thus appearing more risk averse than CRRA would indicate. Moreover, it follows easily that this investor's behavior seems "as if" her preferences exhibit decreasing relative risk aversion.

We now assume that non-market wealth takes the form $z+\widetilde{\varepsilon}$, where $\widetilde{\varepsilon}$ has a mean of zero and is statistically independent of the market risk $\widetilde{r}_{m}$. Since

\footnotetext{
${ }^{6} \mathrm{As}$ it is in the Black-Scholes economy, for example.
} 
$z \neq 0$ makes the investor behave like an investor with HARA preferences ${ }^{7}$, we know from the backgound risk literature that the investor will behave more cautiously in the presence of background risk and purchase a higher proportion of bonds. This follows since HARA preferences satisfy the property of "standard risk aversion" as defined by Kimball (1993). ${ }^{8}$

We now define the derived utility function as $v(x) \equiv E u(x+z+\widetilde{\varepsilon})$. Letting $A_{z, \varepsilon}(x)$ denote the degree of relative risk aversion for the derived utility when $\widetilde{\varepsilon}$ is nondegenerate, we have

$$
A_{z, \varepsilon}(x) \equiv-\frac{x v^{\prime \prime}(x)}{v^{\prime}(x)}=-\frac{x E u^{\prime \prime}(x+z+\widetilde{\varepsilon})}{E u^{\prime}(x+z+\widetilde{\varepsilon})}>-\frac{x u^{\prime \prime}(x+z)}{u^{\prime}(x+z)} \equiv A_{z}(x) .
$$

Comparing $A_{z, \varepsilon}(x)$ to the relative risk aversion for the true utility CRRA function $u$, we see that $A_{z, \varepsilon}(x)>\gamma$, since $A_{z}(x) \geq \gamma$ if $z \leq 0$. In this case, it also follows that $A_{z, \varepsilon}(x)$ is decreasing in $x$, as we show in the appendix (Claim 1).

However, for the case where $z>0$, we have $A_{z}(x)<\gamma$, and thus we cannot compare $A_{z, \varepsilon}(x)$ and $\gamma$ without more information. If $z$ is positive but small, while the $\widetilde{\varepsilon}$ risk is substantial, then the " $\widetilde{\varepsilon}$ effect" will dominate and we will have $A_{z, \varepsilon}(x)>\gamma$. On the other hand, if $z$ is large and $\operatorname{var}(\widetilde{\varepsilon})$ is small, we will obtain just the opposite result. To be more precise, given $x>0$, the essential question is wether $z+\underline{\varepsilon}$ is positive or negative. $\underline{\varepsilon}$ is the minimal value of $\varepsilon$ with positive probability.

First, consider the case $z+\underline{\varepsilon} \geq 0$. Rewriting the previous equation yields

$$
A_{z, \widetilde{\varepsilon}}(x)=E\left[\frac{u^{\prime}(x+z+\widetilde{\varepsilon})}{E u^{\prime}(x+z+\widetilde{\varepsilon})} \frac{-u^{\prime \prime}(x+z+\widetilde{\varepsilon})}{u^{\prime}(x+z+\widetilde{\varepsilon})} x\right]=E^{Q}\left(\frac{\gamma x}{x+z+\widetilde{\varepsilon}}\right)
$$

Hence the relative risk aversion of the derived utility function equals the expectation of $-u^{\prime \prime}(x+z+\widetilde{\varepsilon}) x / u^{\prime}(x+z+\widetilde{\varepsilon})=\gamma x /(x+z+\widetilde{\varepsilon})$ under the riskadjusted probability measure. This is defined by the true probability times

\footnotetext{
7"HARA" refers to "hyperbolic absolute risk aversion," which is fairly common within the finance literature. See, for example, Ingersoll (1987).

${ }^{8}$ See Kimball and Eeckhoudt (1992) for a formal proof. A good survey of this literature can be found in Gollier (2000). One case in which the background risk is not independent of $\widetilde{r}_{m}$ is discussed by Bodie, et al (1992), who assume that the $\widetilde{\varepsilon}$ risk is akin to the risk in some mixture of stocks and bonds.
} 
$u^{\prime}(x+z+\varepsilon) / E u^{\prime}(x+z+\widetilde{\varepsilon})$.

If $z+\underline{\varepsilon} \geq 0$ and $x>0$, then $\gamma x /(x+z+\varepsilon) \leq \gamma$ so that $A_{z, \varepsilon}(x)<\gamma, \forall x>0$. For $x \rightarrow \infty, A_{z, \varepsilon}(x) \rightarrow \gamma$. But this need not imply that $A_{z, \varepsilon}(x)$ monotonically increases in $x$. This is illustrated in Fig. 8a).

Second consider the case $z+\underline{\varepsilon}<0$. Then the previous equation shows that $A_{z, \varepsilon}(x) \rightarrow \infty$ for $x \rightarrow \underline{x}=-z-\underline{\varepsilon}$. Thus, the " $\varepsilon$ - effect" strongly dominates the " $z$ - effect". The " $\varepsilon$ - effect" declines quite rapidly with increasing $x$ so that $A_{z, \varepsilon}(x)$ falls bellow $A(x)=\gamma$ and the " $z$ - effect" dominates. For high values of $x, A_{z, \varepsilon}(x)$ approaches $\gamma$ so that $A_{z, \varepsilon}(x)$ is increasing and concave. This is illustrated in Fig. 8b). These results are summarized in Lemma 1 wich is proved in the appendix.

Lemma 1 Suppose $z>0$ and $z+\underline{\varepsilon}<0$, then:

1. $A_{z, \varepsilon}(x) \rightarrow \infty$ for $x \rightarrow \underline{x}=-z-\underline{\varepsilon}$, and is declining and convex in the range $\left(\underline{x}, x^{o}\right)$.

2. $A_{z, \varepsilon}(x)$ attains a minimum at $x^{o o}>x^{o}$ with $A_{z, \varepsilon}\left(x^{o o}\right)<\gamma$ and increases thereafter.

3. $A_{z, \varepsilon}(x)$ is concave for $x^{o o o}>x^{o o}$.

The lemma shows that the only requirement for the derived relative risk aversion, $A_{z, \varepsilon}(x)$, to start at a high level, decline to some level below $\gamma$ and gradually approach $\gamma$ for high levels of $x$ is that $z+\varepsilon$ can go negative. This is a rather weak requirement which says that non-market wealth has some chance of turning out negative, given a positive $z$. Moreover, initially $A_{z, \varepsilon}(x)$ is convex and later concave so that it starts out declining and convex and eventually becomes increasing and concave. 


\section{The Effect of a Multiplicative Background Risk on Portfolio Demand}

We now consider a multiplicative risk that affects the value of the investor's financial market investment. For example, future tax liabilities might be uncertain; or perhaps money saved for a particular purpose has an unknown future purchasing power. Or consider a case in which the investor wishes to convert her portfolio wealth into a life annuity at date $t=n$ and where the annuity factor is not fully hedgeable. In this case, we consider only the unhedgeable part of the background risk. The investor must now make her portfolio decisions prior to knowing the annuity factor that she will face at date $t=n$.

As is well known under CRRA, if total wealth equals the portfolio of stocks and bonds, multiplying the level of initial wealth by any positive scalar will not change the optimal stock and bond proportions in the portfolio. Suppose that final portfolio wealth is given as $x(\widetilde{R}) \widetilde{y}$, where $\widetilde{y}$ is a random variable with mean one, which is assumed to be statistically independent from $\widetilde{R} .^{9}$ Thus, if $y=1.05$, the final portfolio value is 5 percent higher than expected; or if $y=0.90$ the portfolio value is ten percent lower. Absent any nonmarket wealth, it follows trivially under our assumption of independence that the optimal portfolio choice is not affected by the presence of the random multiplicative background risk $\widetilde{y} .^{10}$ Again, we assume that the investor does not learn about the distribution of $\widetilde{y}$ between dates $t=0$ and $t=n$.

We should caution, however, that this does not imply that the investor is unaffected by the presence of this multiplicative background risk. Indeed, it is trivial to see that $x(\widetilde{R}) \widetilde{y}$ is riskier than $x(\widetilde{R})$ in the sense of Rothschild and Stiglitz (1970), i.e. in the sense of second-degree stochastic dominance. Hence, a risk-averse investor will necessarily be worse off when the $\widetilde{y}$ risk is present. It is just that this investor will not alter her portfolio decision.

\footnotetext{
${ }^{9}$ The assumption of $E \widetilde{y}=1$ implies that we can interpret $(\widetilde{y}-1)$ as a type of unexpected deviation of this multiplicative background risk from its expected value. When this background risk is insignificant, we simply set $\widetilde{y} \equiv 1$.

${ }^{10}$ In a recent paper, Franke, Schlesinger and Stapleton (2006) examine the effect of such multiplicative background risk when we do not have CRRA preferences.
} 
We now examine a portfolio decision in which we have both non-market wealth, $z+\tilde{\varepsilon}$ as well as a multiplicative background risk on the wealth in the investment portfolio. In particular, we consider two different scenarios concerning how the multiplicative risk affects terminal wealth. First, consider the case where the entire wealth is affected by the independent background risk $\widetilde{y}$. In this case, terminal wealth is given as $[x(\widetilde{R})+z+\widetilde{\varepsilon}] \widetilde{y}$. Here we assume that $\widetilde{y}$ and $\widetilde{\varepsilon}$ are mutually independent, in addition to being statistically independent from $\widetilde{R}$. Under our assumption of CRRA, this implies that the investor's objective can be written as either

$$
\max _{x(R)} \frac{1}{1-\gamma} E\left[(x(\widetilde{R})+z+\widetilde{\varepsilon})^{1-\gamma}\right] \cdot E\left(\widetilde{y}^{1-\gamma}\right), \text { for } \gamma>0, \gamma \neq 1
$$

or

$$
\max _{x(R)} E \ln (x(\widetilde{R})+z+\widetilde{\varepsilon})+E \ln \widetilde{y}, \text { for } \gamma=1,
$$

where in either case we are subject to the same budget constraint as in (1). In either of these two cases, the term involving $\widetilde{y}$ is a separable constant and therefore has no effect on the optimal portfolio choice.

The other scenario we consider is for the $\widetilde{y}$ risk to affect only market wealth (i.e. only the assets in the investment portfolio) and not the non-market wealth: $\tilde{w}=x(\widetilde{R}) \widetilde{y}+z+\widetilde{\varepsilon}$. The case where we have both $z=0$ and a degenerate $\widetilde{\varepsilon}($ i.e. $\operatorname{var}(\widetilde{\varepsilon})=0)$ corresponds to the above case with only the multiplicative background risk $\widetilde{y}$.

In the case where $z \neq 0$, but where non-market wealth is not risky $(\operatorname{var}(\widetilde{\varepsilon})=$ 0 ), portfolio choice is affected by the presence of $\widetilde{y}$. In particular, the derived utility in the presence of $z$ alone, $v(x) \equiv u(x+z)$, belongs to the HARA class with relative risk aversion $A_{z}(x)=\gamma x(x+z)^{-1}$. If we now include a nondegenerate multiplicative risk $\widetilde{y}$, the derived utility $v(x) \equiv E u(x \widetilde{y}+z)$ can be either more risk averse or less risk averse than $u(x)$. For example, letting $A_{z, y}(x)$ denote relative risk aversion in this case ${ }^{11}$, if we restrict the wealth level $x$, such that $A_{z}(x y) \geq 1$, i.e. $\gamma \geq 1+\frac{z}{x y} \quad \forall x$ and $\forall y$, it follows from Franke, et al (2006, Corollary 3), that $v(x)$ will be more risk averse than $u(x)$ for $z<0$, i.e. $A_{z, y}(x)>A_{z}(x)>A(x)=\gamma$. Moreover, for $z<0$ relative risk aversion $A_{z, y}(x)$ will be decreasing in $x$, same as $A_{z}(x)$. On

\footnotetext{
${ }^{11} A_{z, y}(x)=E\left[-u^{\prime \prime}(x \tilde{y}+z) \tilde{y}^{2}\right] x / E\left[u^{\prime}(x \tilde{y}+z) \tilde{y}\right]$.
} 
the other hand, for $z>0, v(x)$ will be less risk averse than $u(x)$ for $z>0$, with $A_{z, y}(x)<A_{z}(x)<A(x)=\gamma$. Hence, in the more realistic case with positive fixed non-market wealth, this wealth lowers risk aversion, and the multiplicative background risk also lowers it. However, although $A_{z}(x)$ is increasing in $x, A_{z, y}(x)$ need not also be increasing in $x .^{12}$

The intuition for this result can be obtained from the derived utility function $\nu(x)=E[u(x \widetilde{y}+z)]$. The relative risk aversion of this function is

$$
\begin{aligned}
A_{z, y}(x) & =\frac{-\nu^{\prime \prime}(x)}{\nu^{\prime}(x)} x \\
& =\frac{E\left[-u^{\prime \prime}(x \tilde{y}+z) \tilde{y}^{2}\right]}{E\left[u^{\prime}(x \tilde{y}+z) \tilde{y}\right]} x .
\end{aligned}
$$

Expanding this, we find

$$
\begin{aligned}
A_{z, y}(x) & =E\left[\frac{u^{\prime}(x \tilde{y}+z) \tilde{y}}{E\left[u^{\prime}(x \tilde{y}+z) \tilde{y}\right]} A_{z}(x \tilde{y})\right] \\
& =E\left[A_{z}(x \tilde{y})\right]+\operatorname{cov}\left[\frac{u^{\prime}(x \tilde{y}+z) \tilde{y}}{E\left[u^{\prime}(x \tilde{y}+z) \tilde{y}\right]}, A_{z}(x \tilde{y})\right] .
\end{aligned}
$$

If $z<0$, then relative risk aversion $A_{z}(x y)$ is declining and convex so that $E\left[A_{z}(x \tilde{y})\right]>A_{z}(x)$. If $z>0$, then $A_{z}(x y)$ is increasing and concave so that $E\left[A_{z}(x \tilde{y})\right]<A_{z}(x)$. The assumption that $A_{z}(x y) \geq 1$ assures that $u^{\prime}(x \tilde{y}+z) \tilde{y}$ is declining in $y$. Hence, the covariance term is positive [negative] for $z<[>$ ]0. Intuitively, if the relative risk aversion $A_{z}(x y)$ is convex [concave], then the derived relative risk aversion $A_{z, y}(x)$ is higher [lower] than $A_{z}(x)$.

Consider the above effects in terms of their effect on observed portfolio-choice decisions. If we examine this CRRA investor's portfolio choice, she might appear to be either more or less risk averse than her true level of relative risk aversion $\gamma$. Moreover, her observed behavior might appear to exhibit either increasing or decreasing relative risk aversion.

\footnotetext{
${ }^{12}$ This can be inferred from the Appendix result in Franke et al (2006). It is similar to the observation that increasing absolute risk aversion of the original utility function does not imply increasing absolute risk aversion of the utility function derived over an additive background risk.
} 
If we now allow for a risk $\widetilde{\varepsilon}$ inherent in the non-market wealth, things get more interesting. Given our assumptions, adding the $\widetilde{\varepsilon}$ risk alone always makes the investor behave in a more risk averse manner. We always have $A_{z, \varepsilon}(x)>A_{z}(x)$, for every $x$ and for any level of expected non-market wealth $z$. Of course, with $z>0$, we cannot compare $A_{z, \varepsilon}(x)$ with $A(x)$, since $A_{z}(x)<A(x)$. But the isolated effect of including the riskiness of the nonmarket wealth always makes the individual invest more in bonds. On the other hand, the isolated effect of including the multiplicative background risk $\widetilde{y}$ alone, with a fixed level of non-market wealth depends on the sign of the fixed non-market wealth.

One might think that the combined effect of including both of these independent risks, $\widetilde{y}$ and $\widetilde{\varepsilon}$, simultaneously yields some type of weighted average of these two separate effects - somewhat akin to analyzing income effects together with substitution effects in many comparative-static economic models ${ }^{13}$. However, this is not always the case. The reason for this is somewhat subtle.

Consider first the case where $z \leq 0$. If there is non-market wealth risk, then $A_{z, \varepsilon}(x)>A(x)$ and $A_{z, \varepsilon}(x)$ is declining and convex as shown in the appendix (Claim 2). Hence $A_{z, \varepsilon}(x)$ has a similar pattern as $A_{z}(x)$ in case of $z<0$ and $\operatorname{var}(\tilde{\varepsilon})=0$. For the latter case, we have seen in the previous section that the multiplicative risk raises risk aversion. The same is true in case of $z \leq 0$ and $\operatorname{var}(\tilde{\varepsilon})>0$, provided that $A_{\varepsilon}(x) \geq 1$. This follows again from Franke et al (2006, Corollary 1(i)). Hence both background risks reinforce each other ${ }^{14}$ : $A_{\varepsilon, y}(x)>A_{\varepsilon}(x)>A(x)=\gamma$.

In the absence of the multiplicative risk, $A_{\varepsilon}(x)$ is declining. This property still holds in the presence of both risks: $A_{\varepsilon, y}(x)$ is declining (see the appendix in Franke et al (2006)).

\footnotetext{
${ }^{13}$ It is important to note that even though the additive and the multiplicative risk are, by assumption, independent, it is not feasible to use a derived utility function, derived only over different outcomes of the multiplicative risk $y$. More precisely, given wealth $x \tilde{y}+z+\varepsilon$, defining $E[u(x \tilde{y}+z+\varepsilon) \mid x, \varepsilon] \equiv v(x+z+\varepsilon)$ is wrong because $v(x+z+\varepsilon)$ suggests that the agent is indifferent to a change in $x$ and $(z+\varepsilon)$ which preserves the sum $x+z+\varepsilon$. This is wrong because $x$ is subject to the $y$-risk, in contrast to $(z+\varepsilon)$.

${ }^{14} A_{z, \varepsilon, y}(x)=E\left[-u^{\prime \prime}(x \tilde{y}+z+\tilde{\varepsilon}) \tilde{y}^{2}\right] x / E\left[u^{\prime}(x \tilde{y}+z+\tilde{\varepsilon}) \tilde{y}\right]$. $A_{\varepsilon, y}(x)=A_{z, \varepsilon, y}(x)$ for $z=0$.
} 
The intuition for these results is that the non-market wealth risk makes the agent feel "poorer". This is seen by considering Kimball's precautionary premium for the non-market wealth risk $\tilde{\varepsilon}$. This premium is positive and declines in increasing $x$. Thus, its effect on risk aversion is similar to the effect of a negative fixed non-market wealth. Hence, a multiplicative background risk raises risk aversion in both cases.

Given that, it is not surprising that these effects are even more pronounced for negative fixed non-market wealth. Let $A_{z, \varepsilon, y}(x)$ denote the relative risk aversion in the presence of both background risks and a fixed non-market wealth. Then we obtain Proposition 1 which is proved in the appendix.

Proposition 1 Suppose that the investor has a utility function with constant relative risk aversion $\gamma \geq 1$. Then for nonpositive fixed non-market wealth, $z \leq 0$

a) $A_{z, \varepsilon, y}(x)>A_{z, \varepsilon}(x)>A_{z}(x)>A(x)=\gamma$

b) $A_{z, \varepsilon, y}(x), A_{z, \varepsilon}(x), A_{z, y}(x)$ and $A_{z}(x)$ are declining.

By Proposition 1, we can easily predict the effects of background risks, given a nonpositive fixed non-market wealth. The reason is that all three background-variables $(z, \varepsilon, y)$ act in a similar manner. All three backgroundvariables make the investor act more cautiously. When her marketable wealth $x$ increases, she feels less threatened by these background-variables. Therefore she acts in a less cautious manner. This appears very intuitive.

The situation is more complicated in the case of positive fixed non-market wealth, $z>0$. The non-market wealth risk still raises observed risk aversion in the absence of the multiplicative risk, but it produces a U-shaped relative risk aversion pattern if non-market wealth is negative with positive probability (Figure 8b). The multiplicative risk, in contrast, lowers observed relative risk aversion in the absence of the non-market wealth risk. Thus, we would expect that both background risks together would mitigate each other so that observed risk aversion is closer to $A(x)=\gamma$. This, however, is not generally true. It may be that $A_{z, \varepsilon, y}(x)>A_{z, \varepsilon}(x)>A_{z}(x)$, but also $A_{z, \varepsilon, y}(x)<A_{z, \varepsilon}(x)$ is possible. 
Critical for the effect of the multiplicative risk on observed risk aversion is how $A_{z, \varepsilon}(x y)$ behaves. Assume $A_{z, \varepsilon}(x y) \geq 1$. In the previous section we have seen that the effect on relative risk aversion of the multiplicative risk depends on whether relative risk aversion $A_{z}(x y)$ is convex or concave. Now, with nonmarket wealth risk, the same reasoning applies to $A_{z, \varepsilon}(x y)$. Hence it is crucial whether $A_{z, \varepsilon}(x y)$ is convex or concave. Assume that non-market wealth is negative with positive probability. Then, as shown in Lemma $1, A_{z, \varepsilon}(x)$ is convex (and declining) for low values of $x$, but concave (and increasing) for high values of $x$. Therefore, it is not surprising that for low values of $x$ the multiplicative risk raises relative risk aversion instead of lowering it, as it does in the absence of the $\varepsilon$ risk. The $\varepsilon$ risk induces a switch from an increasing, concave relative risk aversion, $A_{z}(x)$ to a declining, convex relative risk aversion $A_{z, \varepsilon}(x)$. This reverses the direction of the effect of a multiplicative risk from lowering to raising the derived relative risk aversion. Hence both background risks aggravate each other, in a manner similar to that in the case of a negative expected non-market wealth. For large values of $x, A_{z, \varepsilon}(x)$ is increasing and concave, as in the absence of the $\varepsilon$ risk. Then the multiplicative risk lowers $A_{z, \varepsilon, y}(x)$ below $A_{z, \varepsilon}(x)$. But in between there is also a range of $x$ in which neither condition holds. Therefore we cannot predict wether $A_{z, \varepsilon, y}(x)$ will be higher or smaller than $A_{z, \varepsilon}(x)$ in this range.

These results are formally stated in Proposition 2.

Proposition 2 Suppose that the investor has a CRRA utility function with positive fixed non-market wealth $z$ and relative risk aversion $A_{z, \varepsilon}(x y) \geq 1$. Then

a) $A_{z, \varepsilon, y}(x)>A_{z, \varepsilon}(x)$ if $A_{z, \varepsilon}(x y)$ is declining and convex,

b) $A_{z, \varepsilon, y}(x)<A_{z, \varepsilon}(x)$ if $A_{z, \varepsilon}(x y)$ is increasing and concave $e^{15}$.

We illustrate all of the potential effects above with an example in the following section.

\footnotetext{
${ }^{15}$ The proof of Proposition 2a) follows directly from Franke et al (2006, Corollary 1(i)), while the proof of Proposition 2b) follows directly from their Corollary 2(i).
} 


\section{Investment Simulations}

Our simulations are based around twelve different scenarios, detailed in Table 1. As we have seen above, the derived risk aversion of an agent and the resulting optimal portfolio demand depends upon three factors. The expected level of non-market wealth, $z$, the additive background risk associated with it, and the multiplicative background risk which attaches to market plus non-market wealth. We assume in Table 1, that expected non-market wealth can be zero (the base case), positive (the most likely case) or negative. The additive background risk, measured by the standard deviation of non-market wealth, $\sigma_{\varepsilon}$, can be positive or zero. The multiplicative background risk, measured by the standard deviation of the rollover rate, $\sigma_{y}$, can also be positive or zero. Since all combinations are possible, there are a total of twelve possible cases.

In order to illustrate the effects of non-market wealth on portfolio choice, we now present numerical examples for each of the cases above. In Table 2 we summarize the data on which the numerical simulations are based. We assume that there are seven years with portfolio purchases made at dates $, t=0,1, \ldots, 6$ and terminal wealth realized at date $t=7$. The random values for $\widetilde{\varepsilon}$ and $\widetilde{y}$ are also only realized at date $t=7$. We thus have a static decision problem, with portfolio rules set at date $t=0$. We use this multi-period set-up because the presence of the $\widetilde{\varepsilon}$ and $\widetilde{y}$ risks not only affect the initial portfolio choice, but they also affect the intertemporal strategy for portfolio rebalancing. ${ }^{16}$

We approximate the geometric Brownian motion for the risky market return using a binomial approximation, where the mean excess return over any year is 5\%. A risk-free one-year maturity bond pays $5 \%$ over each year. The annualized volatility of the market return is $20 \%$. The investor has an initial wealth of 100, and has CRRA preferences with a coefficient of relative risk aversion, $\gamma=1.5$. In the various cases shown below, the expected value of the non-market wealth, $z$ takes on values of $-20,0$ and 30 .

\footnotetext{
${ }^{16}$ Of course, a truly dynamic model, with learning about $\widetilde{\varepsilon}$ and $\widetilde{y}$ would be more realistic, but also more complex. Our point here is to show how these risks can affect observed portfolio-choice behavior, even in this simple setting.
} 
The $\widetilde{\varepsilon}$ risk in our simulation is calculated by $\widetilde{\varepsilon}$ taking on a binomial distribution with $n=4$ and $p=0.5$, and where each "success" is modelled as an increase in $\widetilde{\varepsilon}$ by 15 , whereas "failure" is modelled as a decrease in $\widetilde{\varepsilon}$ by 15 . Hence, in the presence of the $\widetilde{\varepsilon}$ risk non-market wealth is always negative with positive probability. The background risk $\widetilde{y}$ also is binomial with $n=1$ and $p=0.5$, with the initial value of $\widetilde{y}$ set at one, and with "success" indicating a 30 percent increase in $\widetilde{y}$, whereas "failure" indicates a 30 percent decrease in $\widetilde{y}$.

\subsection{Fixed (Non-Stochastic) Non-Market Wealth}

In Figure 1 we illustrate the optimal solution using the log-demand function from equation (5). The optimal demand function is computed by solving the first order condition for each state at date $n$, subject to the budget constraint embedded in (1). In case 1.1, the expected non-market wealth is $z=0$. With $z=0$, both the relative risk aversion $A(x)$ is a constant and the elasticity of the pricing kernel $\left(-\frac{d \ln \phi}{d \ln R}\right)$ in (5) is also a constant given the geometric Brownian motion generating the market return. Thus, the optimal log-demand for state contingent claims is linear. This is an example of the Merton case.

Case 1.2 shows the effect of a positive non-market wealth. Here we assume that $z=30$. The resulting optimal demand function is obtained by substituting $A_{z}(x)$ for $A(x)$ in (5). The demand function is steeper, reflecting the lower derived relative risk aversion. It is also concave reflecting the fact that, in this case, relative derived risk aversion is increasing. Case 1.3, shows the effect of a negative expected non-market wealth, where $z=-20$. In this case, the demand curve is less steep and convex, reflecting greater risk aversion as well as decreasing relative risk aversion for the derived utility.

Also, we should point out that it is not by chance that the demand functions for contingent claims cross at one point in the diagram. In the state of the world where the realized market return equalled the risk-free return in all five periods, i.e. $R=\left(1+r_{f}\right)^{5}=\left[(1.05)^{5}\right]$, every investment strategy will yield the same terminal wealth .

Using these cases as a starting point, we next examine the effect of either 
adding risk to non-market wealth, or adding a multiplicative background risk, or both.

\subsection{Background Risks}

In order to delineate the cases, we consider three alternative scenarios in the presence of background risks: namely the cases where the expected net non-market wealth is zero, negative or positive.

\subsubsection{Zero expected non-market wealth}

First consider the case where the expected non-market wealth is zero, $z=0$. As mentioned previously, adding the background risk $\widetilde{y}$ in this case has no effect on portfolio choice. This is seen in Figure 2 by noting that the base case (with $\widetilde{\varepsilon} \equiv 0$ ) in case 2.1 yields identical results to case 2.2 .

Now consider adding risk $\widetilde{\varepsilon}$ to the non-market wealth. If the realized value of $\widetilde{\varepsilon}$ is zero, the individual's net position in non-market wealth is zero: any assets perfectly offset any liabilities. If $\widetilde{\varepsilon}$ is positive, the individual has a unexpected net positive value of non-market wealth, whereas a negative value for $\widetilde{\varepsilon}$ corresponds to a negative net position in non-market wealth. This allows us to focus on pure risk effects. As is known in this case, the individual behaves in a more risk-averse manner when the non-market wealth is risky. This is seen in Figure 2 by comparing the base case (with $\widetilde{\varepsilon} \equiv 0$ ) in case 2.1 with the case of a positive $\widetilde{\varepsilon}$ risk in case 2.3 . In this case, the demand for state claims is flatter, due to the more-risk-averse portfolio choice. Moreover, the demand curve is no longer linear, but rather convex, due to the decreasing relative risk aversion in this case. ${ }^{17}$

If we now consider both the $\widetilde{\varepsilon}$ risk and $\widetilde{y}$ risks as existing concurrently, it follows from the appendix that behavior will be even more risk averse than under the $\widetilde{\varepsilon}$ risk alone in the case where relative risk aversion is one or higher, $\gamma \geq 1$. Although the $\widetilde{y}$ risk in isolation does not affect behavior, it makes the

\footnotetext{
${ }^{17}$ As $\ln R$ increases, the slope $\frac{d \ln x}{d \ln R}$ increases, due to the lower level of $A_{z, \varepsilon}$ in (5). See the appendix for a proof.
} 
investor worse off and here we see how it causes the investor to behave as if she were poorer and hence more sensitive to the additional $\widetilde{\varepsilon}$ risk. Moreover, it follows in this case that $v(x) \equiv E u(x \widetilde{y}+\widetilde{\varepsilon})$ exhibits decreasing relative risk aversion. ${ }^{18}$ This is illustrated in case 2.4 of Figure 2.

\subsubsection{Negative expected non-market wealth}

When $z<0$, behavior appears to be more risk averse than CRRA would indicate as well as seeming to exhibit decreasing relative risk aversion. Adding the background risk $\widetilde{y}$ makes behavior seem even more risk averse. This shows up only slightly in Figure 3 in cases 3.1 and 3.2.

It also follows in this case that adding the $\widetilde{\varepsilon}$ risk to $z$, but in the absence of the $\widetilde{y}$ risk, makes behavior seem more risk averse and also seem to exhibit decreasing relative aversion, as is seen by comparing cases 3.1 and 3.3 in Figure 3.

If we include both the $\widetilde{\varepsilon}$ risk and the $\widetilde{y}$ risk simultaneously, we see that the effects of more-risk-averse behavior and of decreasing relative risk aversion are magnified. Case 3.4 in Figure 3 illustrates this situation.

\subsubsection{Positive expected non-market wealth}

This case is quite intriguing. When $z>0$, but background risks are absent, the individual appears to behave in a less risk-averse manner than CRRA would indicate. In addition, behavior appears to exhibit increasing relative risk aversion. This is the base case 4.1 in Figure 4, where the log-demand curve for contingent clams is slightly concave. If we add the background multiplicative risk $\widetilde{y}$, behavior here becomes slightly less risk averse, as indicated by the slightly steeper demand curve, as illustrated in case $4.2 .{ }^{19}$

\footnotetext{
${ }^{18}$ This follows by noting that $\widehat{v}(x) \equiv E u(x+\widetilde{\varepsilon})$ satisfies decreasing relative risk aversion, as shown in the appendix of this paper. Hence, from Franke, et al (2006, appendix), it follows that adding the $\widetilde{y}$ risk to obtain $v(x) \equiv E u(x \widetilde{y}+\widetilde{\varepsilon})$ preserves this property.

${ }^{19}$ The property of increasing relative risk aversion need not always hold in this setting, but it does in this example.
} 
If we omit the multiplicative $\widetilde{y}$ risk, but add a risk $\widetilde{\varepsilon}$ to the non-market wealth, the addition of $\widetilde{\varepsilon}$ increases the level of risk aversion. Since non-market wealth is negative with positive probability, Lemma 1 applies. Hence, for low levels of marketable wealth relative risk aversion is higher than $\gamma$, then it declines to a level below $\gamma$ and gradually approaches $\gamma>0$ for high levels of marketable wealth. Hard to see in the diagram for case 4.3, but for low values of $\ln R$ the log-demand curve for contingent claims is convex, indicating decreasing relative risk aversion. But at the same time, for high levels of $\ln R$, the demand curve is slightly concave, indicating that relative risk aversion is increasing.

In case 4.4 , we show the effects of including both the $\widetilde{\varepsilon}$ risk and the $\widetilde{y}$ risks simultaneously. Although the effect of the $\widetilde{y}$ risk in isolation is to cause a decrease in observed risk aversion, the $\widetilde{y}$ risk also makes the individual more sensitive to the $\widetilde{\varepsilon}$ risk. From Lemma 1 and Prop. 2 we know that for low levels of marketable wealth both risks reinforce each other making behavior appear more risk averse than in the presence of the $\widetilde{\varepsilon}$ risk only. This is evident in Fig. 4 from the small slope of the 4.4-curve in the range of low market returns. For high levels of marketable wealth, the $\widetilde{y}$ risk makes behavior appear less risk averse than in the presence of the $\widetilde{\varepsilon}$ risk only. Hence in Fig. 4 the slope of the 4.4-curve is smaller than that of the 4.2-curve in the range of high market returns.

\section{Stock Proportions and Dynamic Allocation}

Given our assumptions that the market follows a geometric Brownian motion and that a riskless bond exists, the market is dynamically complete as in the Black-Scholes world. It follows that any state-contingent claim demand $x(R)$ can be replicated with a period-by-period stock/bond strategy. In the case where $A(x)$ is a constant, we know from Merton (1971) that the replicating strategy is to hold a constant proportion of wealth in stocks, throughout the period from time 0 to time $n$. However, in the general case, with derived utility $v(x)=E u(x \widetilde{y}+z+\widetilde{\varepsilon})$, the measure of relative risk aversion for $v$, $A_{z, \varepsilon, y}(x)$, is not constant. Hence, the dynamic strategy is more complex. 
In the following numerical simulations, we approximate the market return with a log-binomial process. At time $n$, the state-contingent claim $x(R)$ has $n+1$ outcomes, indexed by $i=0,1, \ldots, n$. Moving back to time $n-1$, the market return has $i=0,1, \ldots, n-1$ states. In state $i$ at time $n-1$, the market return can only move to state $i$ or state $i+1$ at time $n$. It follows that there is a unique stock/bond strategy for each state at time $n-1$. The optimal dynamic strategy can be found by moving back through the binomial tree and solving for the stock/bond proportions at each point of time and in each state. If $r_{m, t}$ is the market return in period $t$, and the risk-free rate is $r_{f}$, then

$$
\frac{x}{x_{0}}=\Pi_{t=1}^{n}\left[1+\alpha_{i, t-1} r_{m, t}+\left(1-\alpha_{i, t-1}\right) r_{f}\right]
$$

is solved for the dynamic stock proportion, $\alpha_{i, t}$.

Table 3 shows the optimal stock proportion in year 0 and year 6 , across different states, for the twelve different cases illustrated previously in Figures 1-4. The year- 6 states are indexed by the number of down-ticks of the binomial process of the market return. Hence, state 0 is the highest market state and state 6 is the lowest. From (5), the percentage of stocks in the portfolio reflects the degree of relative risk aversion across the various states. Hence, if the percentage is constant (declining) (increasing) across states, this indicates constant (declining) (increasing) derived relative risk aversion for market wealth. The results are shown for all the twelve different cases, which allow us to analyze the effects of the expected value of non-market wealth and its risk $\widetilde{\varepsilon}$ and the the multiplicative $\widetilde{y}$ risk, both separately and jointly.

Cases 1-4 show the effect of the two risks in the case of a zero-mean nonmarket wealth, $z=0$. In the absence of both the non-market wealth risk and the multiplicative background risk, the investor follows the Merton strategy, investing $78 \%$ of her wealth in stocks in year 0 and also $78 \%$ in each state at year 6 . When multiplicative background risk $\widetilde{y}$ risk alone is introduced (case 2 ), there is no effect on stock proportions. This is due to the fact that the utility is CRRA and $z=0$. The introduction of additive non-market wealth risk (case 3), reduces stock proportions and causes the proportions in year 6 to be state dependent, reflecting the convexity of the log-return function. The investor behaves towards the market risk like someone with declining relative risk averse (DRRA) utility. This in turn implies that the further 
introduction of the background $\widetilde{y}$ risk makes the investor choose even less stocks (case 4).

Cases 5-8 show the effect of the two risks individually and jointly in the case where the expected non-market wealth is negative, $z<0$. In all four cases the investors behavior is consistent with DRRA. This is illustrated by the stock proportions in year 6 , which are higher in the high market states. Also, the effect of the non-market wealth and $\widetilde{y}$ risks is straightforward in this set of cases. Non-market wealth risk $\widetilde{\varepsilon}$ alone reduces stock proportions, as does $\widetilde{y}$ risk. Also, the joint effect of the two risks together (case 8) is to reduce stock proportions even more.

The more complex and perhaps more relevant scenarios are illustrated in cases $9-12$, where the expected value of non-market wealth is positive, $z>$ 0 . Here, in case 9, where both risks are zero, observed behavior exhibits increasing relative risk aversion, IRRA. In case 10, where the multiplicative background risk $\widetilde{y}$ is introduced, the effect is to increase the investment in stocks, both in year 0 and in year 6 . In this example, IRRA behavior is preserved under the $\widetilde{y}$ risk. However, the effect of introducing the non-market wealth risk $\widetilde{\varepsilon}$ alone, in case 11 , is to reduce the stock investment and, given the chosen parameter values, to produce the U-shaped behavior for relative risk aversion as mentioned in the previous section. This is because the effect of the non-market wealth risk and the consequent precautionary premium outweigh the effect of the positive expected non-market wealth on the utility function.

This also explains why, in case 12 , the compounding effect of the multiplicative $\widetilde{y}$ risk now reduces the stock proportion even further in the low states. However, in the high states, the multiplicative risk now increases the investment in stocks. This is because in the high states the precautionary premium for the $\widetilde{\varepsilon}$ risk is very small. It is important to note however that the effects on derived relative risk aversion exhibited here depend upon the positive probability of a negative non-market wealth. If non-market wealth is always positive, then the effects on derived relative risk aversion are quite different.

The examples shown in Table 4 emphasize this point using sensitivity analysis on the parameter values. Cases $11 \mathrm{a}$ and $11 \mathrm{~b}$ and $12 \mathrm{a}$ and $12 \mathrm{~b}$ show the effect 
of varying the size of the additive risk, $\sigma_{\varepsilon}$. 11a and $12 \mathrm{a}$ show the effect on example 11 of lower and higher $\sigma_{\varepsilon}$ respectively. Note that the lower risk in 11a induces IRRA derived utility. The higher risk in 11b induces DRRA derived utility. These cases are in contrast to case 11, where derived relative risk aversion is U-shaped. The corresponding response to the multiplicative risk is shown in case 12a, where all stock proportions are higher than in the absence of the multiplicative risk (case 11a) and in case 12b, where all stock proportions are lower than in the absence of the multiplicative risk (case $11 b)$.

A further sensitivity analysis is carried out with respect to the coefficient of relative risk aversion $(\gamma)$. 11c and $12 \mathrm{c}$ show that the effects are preserved, but dampened in the case of higher risk aversion. $11 \mathrm{~d}$ and $12 \mathrm{~d}$ show that the effects are preserved, but enhanced in the case of lower risk aversion.

In the figures 5-7, we graphically illustrate the optimal asset allocation strategy for a few of these cases over a five-year time interval. In Figure 5, we assume that expected non-market wealth is $z=30$, non-market wealth risk $\widetilde{\varepsilon}=0$ and the multiplicative risk $\widetilde{y}$ is distributed as discussed in the preceding section. This is case 10, in Table 3. The effect of the positive-mean nonmarket wealth is to produce less risk averse behavior that exhibits IRRA and this is reflected in the dynamic asset allocation strategy shown. The investor starts with $96 \%$ invested in stocks at year 0 . Then at year 1, this falls to $92 \%$ if the market moves up (0 down moves) and increases to $99 \%$ if the market moves down (1 down move). At year 2, the investor puts either 90\%, 95\% or $103 \%$ in stocks depending on the market state. Since there is an inverse relationship between the number of down-moves and the level of the market, the strategy reflects IRRA utility (more is invested in stocks as the market declines).

In Figure 6, we assume that expected non-market wealth is positive, $z=0$, the non-market wealth is risky $(\widetilde{\varepsilon}=0)$ and the multiplicative background risk $\widetilde{y}$ is as defined in the previous section. This is case 4, in Table 3. The effect is to produce IRRA behavior and this is reflected in the dynamic asset allocation strategy shown. The investor starts with $55 \%$ invested in stocks at year 0 . Then at year 1 , this increases to $61 \%$ if the market moves up ( 0 down moves) and declines to $50 \%$ if the market moves down (1 down move). At year 2 , the investor puts either $65 \%, 55 \%$ or $44 \%$ in stocks depending on the 
market state. Since there is an inverse relationship between the number of down-moves and the level of the market, the strategy reflects DRRA utility (less is invested in stocks as the market declines).

In Figures 7 and 8, we illustrate the optimal dynamic strategy for cases 11 and 12 in Table 3 . Here, the positive $z$ is balanced by a positive nonmarket wealth risk. In Figure 7 there is no multiplicative risk. In Figure 8 the binomial distribution of $\tilde{y}$ is assumed. Figure 7 reveals an interesting pattern of stock proportions. The $0 d$ curve shows that as the market rises, the investor first invests more in stocks (DRRA) but then reduces it in later years (IRRA). Similar patterns are reflected in Figure 8.

The resulting outcome shows an inverted U-shape allocation strategy. As the market starts to fall, the investor invests more in stocks at first. But if the market continues falling, she starts to decrease her investment in stocks. This phenomenon follows by examining relative risk aversion at the appropriate wealth levels. Relative risk aversion is U-shaped here, and we are initially on the upward sloping part of the "U." If the market rises, observed behavior seems to exhibit increasing relative risk aversion (a lower percent in stock as wealth increases). However for downward movement in the market, risk aversion initially falls but then rises, as we pass the trough on the U-shape relative risk aversion.

\section{Conclusions}

Portfolio selection is complicated by personal circumstances which can radically affect the asset allocation strategy of the investor. Here, we have analyzed the optimal strategy of a CRRA investor in a market where a single risky asset follows a geometric Brownian motion. The investor has stochastic non-market wealth and also a multiplicative background risk and chooses investment strategies. If we only observe the portfolio choice of the investor, it might be difficult to observe anything that looks similar to her underlying CRRA preferences. The existence of non-market wealth may cause the investor to act as if her utility had increasing or declining relative risk aversion, depending on the size and risk of the non-market wealth. The response to 
a multiplicative background risk crucially depends upon the nature of any non-market wealth and its riskiness .

Consideration of the additive non-market wealth risk and the multiplicative background risk together in one model is important, since the combined effect can be quite different from the effect of one of these risks alone. Consider the case in which non-market wealth has positive expectation. Then the effect of the multiplicative $\widetilde{y}$ risk alone is to increase investment in stocks, whereas the effect of this same $\widetilde{y}$ risk may be to reduce investment in stocks when non-market wealth risk $\widetilde{\varepsilon}$ already exists and non-market wealth can turn out negative. Ignoring the interaction effects between the risks can lead to incorrect predictions.

In our model, resolution of the uncertainty surrounding the non-market wealth risk and multiplicative background risks only takes place at the horizon date. We solve what is essentially a single-period model for the optimal demand function for state-contingent claims. However, since the market for the risky asset is dynamically complete, this function can be represented by a dynamic asset-allocation strategy involving stocks and bonds. We find that this strategy is both time and state dependent. It follows that simple prescriptions for asset-allocation such as "lifestyle", which suggests a shift of assets from stocks to bonds as retirement approaches, is unlikely to be optimal. 


\section{Appendix}

\section{Proof of Lemma 1}

By definition,

$$
\begin{aligned}
A_{z, \varepsilon}(x) & =\frac{E-u^{\prime \prime}(x+z+\varepsilon)}{E u^{\prime}(x+z+\varepsilon)} x \\
& =E\left[\frac{u^{\prime}(x+z+\varepsilon)}{E u^{\prime}(x+z+\varepsilon)} \frac{-u^{\prime \prime}(x+z+\varepsilon)}{u^{\prime}(x+z+\varepsilon)}\right] x \\
& =\gamma\left[\frac{u^{\prime}(x+z+\varepsilon)}{E u^{\prime}(x+z+\varepsilon)} \frac{x}{x+z+\varepsilon}\right] .
\end{aligned}
$$

Hence, $A_{z, \varepsilon}(x) \rightarrow \infty$ for $x \rightarrow \underline{x}=-\underline{\varepsilon}-z$. $\underline{\varepsilon}$ is the minimal value of $\varepsilon$ with positive probability (density). Also, $A_{z, \varepsilon}^{\prime}(x) \rightarrow-\infty$ for $x \rightarrow \underline{x}$. Since $A_{z, \varepsilon}(x)>0$ for $x>\underline{x}, A_{z, \varepsilon}^{\prime \prime}(x)>0$ is implied for some range $x \in\left(\underline{x}, x^{\circ}\right)$ with $x^{\circ}>\underline{x}$.

Next, we show that $A_{z, \varepsilon}(x)$ is increasing for large values of $x$. This implies that there exists a finite $x^{\circ \circ}$ at which $A_{z, \varepsilon}(x)$ attains a minimum.

Given a CRRA-utility function,

$$
\begin{aligned}
A_{z, \varepsilon}(x) & =\gamma \frac{E(x+z+\varepsilon)^{-\gamma-1}}{E(x+z+\varepsilon)^{-\gamma}} x \\
& =\gamma \frac{[x+z-\varphi(\varepsilon \mid x)]^{-\gamma-1}}{[x+z-\varphi(\varepsilon \mid x)]^{-\gamma}}\left(1-\frac{\partial \varphi}{\partial x}\right) x
\end{aligned}
$$

with $\varphi(\varepsilon \mid x)$ being Kimball's (1990) precautionary premium of $\varepsilon$ given $x$. Since we now consider large values of $x$, we may regard $\varepsilon$ as a small risk in the sense of Pratt (1964). Technically, divide $x+z+\varepsilon$ by a large positive constant $c$ so that $\sigma(\varepsilon / c)$ is a small risk. Then, dropping $c$ for notational simplicity, for a large $x$ the precautionary premium is given by [see Pratt (1964)]

$$
\varphi(\varepsilon \mid x)=\frac{\gamma+1}{x+z} \frac{\sigma^{2}(\varepsilon)}{2}
$$


so that

$$
\frac{\partial \varphi}{\partial x}=-\frac{\varphi(\varepsilon \mid x)}{x+z}
$$

Hence,

$$
\begin{aligned}
A_{z, \varepsilon}(x) & =\gamma \frac{1+\varphi(\varepsilon \mid z) /(x+z)}{x+z-\varphi(\varepsilon \mid x)} x \\
& =\gamma \frac{x}{x+z} \frac{x+z+\varphi(\varepsilon \mid x)}{x+z-\varphi(\varepsilon \mid x)} \\
& =\gamma \frac{x}{x+z} \frac{1+\frac{\gamma+1}{2} \sigma^{2}(\varepsilon)(x+z)^{-2}}{1-\frac{\gamma+1}{2} \sigma^{2}(\varepsilon)(x+z)^{-2}}
\end{aligned}
$$

For large values of $x$, the second fraction converges much faster to 1 than the first fraction because the second fraction depends on $(x+z)^{-2}$. Therefore, $A_{z, \varepsilon}(x) \rightarrow \gamma \frac{x}{x+z}<\gamma$ and finally to $\gamma$. Hence, $A_{z, \varepsilon}(x)$ is increasing for large values of $x$.

Finally, in the last equation the first fraction is concave in $x$ while the second is convex. Again, for high values of $x$, the first fraction "dominates" the second, which moves much faster to 1 . Therefore, there exists some $x^{000}>x^{00}$ so that $A_{z, \varepsilon}(x)$ is concave in $x$ for $x>x^{\circ 00}$. 
Claim 1: Let $z \leq 0$ and $v(x)=E u(x+z+\widetilde{\varepsilon})$, where $u$ is CRRA. Then $v$ exhibits decreasing relative risk aversion.

Proof: Define $\widehat{v}(x) \equiv u(x+z)$. Then $\widehat{v}(x)$ exhibits standard risk aversion and either constant or decreasing relative risk aversion. We let $A_{z}(x)$ denote the relative risk aversion for $\widehat{v}$. Now

$$
v^{\prime}(x)=E \widehat{v}^{\prime}(x+\widetilde{\varepsilon}) \equiv \widehat{v}^{\prime}(x-\psi(x)),
$$

where $\psi(x)$ denotes Kimball's (1990) precautionary premium for $\widetilde{\varepsilon}$.

Relative risk aversion for $v(x)$ is then easily calculated as

$$
A_{z, \varepsilon}(x)=-\frac{x \widehat{v}^{\prime \prime}(x-\psi(x))\left(1-\psi^{\prime}(x)\right)}{\widehat{v}^{\prime}(x-\psi(x))}=A_{z}(x-\psi) \cdot \frac{\left(1-\psi^{\prime}(x)\right)}{1-\frac{\psi}{x}} .
$$

Since $\widehat{v}$ exhibits standard risk aversion, we know from Kimball (1991) that $\psi^{\prime}(x)$ is negative. Hence (7) implies $1-\frac{\psi}{x}>0$.

Straightforward calculation from (7) shows that

$$
\begin{gathered}
A_{z, \varepsilon}^{\prime}(x)=A_{z}^{\prime}(x-\psi) \cdot \frac{\left(1-\psi^{\prime}\right)^{2}}{1-\frac{\psi}{x}} \\
+A_{z}(x-\psi) \cdot \frac{\left(-\psi^{\prime \prime}\right)\left(1-\frac{\psi}{x}\right)+\left(1-\psi^{\prime}\right)\left(\frac{x \psi^{\prime}-\psi}{x^{2}}\right)}{\left(1-\frac{\psi}{x}\right)^{2}} .
\end{gathered}
$$

The first term on the right-hand side in (8) is non-positive by the assumptions. The second term is negative since $\psi^{\prime \prime}>0$, which follows from Franke et al (1998, Lemma 2). Hence, $A_{z, \varepsilon}^{\prime}(x)<0$ 
Claim 2: Let $z \leq 0$ and $v(x)=E u(x+z+\widetilde{\varepsilon})$, where $u$ is CRRA. Then $v$ exhibits convex relative risk aversion.

First, we prove

Lemma 2 If $u$ is a HARA-utility function with $\gamma>0$, then $\varphi^{\prime}(x)<0, \varphi^{\prime \prime}(x)>$ 0 and $\varphi^{\prime \prime \prime}(x)<0$.

Proof: Franke et al (1998) have shown $\varphi^{\prime}(x)<0$ and $\varphi^{\prime \prime}(x)>0$. Therefore $\varphi^{\prime \prime \prime}(x)<0$ remains to be shown. For notational convenience, let $\nu=x+z$ and $\tilde{\varepsilon}=\sigma \tilde{\eta}$, where $\tilde{\eta}$ is a random variable with mean zero and unit variance. We have

$$
(\nu-\psi)^{-\gamma}=E\left[(\nu+\tilde{\varepsilon})^{-\gamma}\right]
$$

or

$$
\left(1-\frac{\psi}{\nu}\right)^{-\gamma}=E\left[\left(1+\frac{\sigma \tilde{\eta}}{\nu}\right)^{-\gamma}\right]
$$

For a given $\eta$-distribution, it follows that

$$
\frac{\psi}{\nu}=f\left(\frac{\sigma}{\nu}\right)
$$

or

$$
\psi=\nu f\left(\frac{\sigma}{\nu}\right) .
$$

Differentiating w.r.t. $\nu$

$$
\begin{aligned}
\psi_{\nu} & =f\left(\frac{\sigma}{\nu}\right)+\nu f^{\prime}\left(\frac{\sigma}{\nu}\right) \frac{-\sigma}{\nu^{2}} \\
& =f\left(\frac{\sigma}{\nu}\right)-f^{\prime}\left(\frac{\sigma}{\nu}\right) \frac{\sigma}{\nu}
\end{aligned}
$$

and differentiating w.r.t. $\sigma$

$$
\psi_{\sigma}=\nu f^{\prime}\left(\frac{\sigma}{\nu}\right) \frac{1}{\nu}=f^{\prime}\left(\frac{\sigma}{\nu}\right)
$$

Hence

$$
\psi_{\nu}=f\left(\frac{\sigma}{\nu}\right)-\psi_{\sigma} \frac{\sigma}{\nu}
$$


Differentiating again w.r.t. $\nu$

$$
\psi_{\nu \nu}=\frac{\partial \psi_{\nu}}{\partial \frac{\sigma}{\nu}} \frac{\partial \frac{\sigma}{\nu}}{\partial \nu}=\frac{\partial \psi_{\nu}}{\partial \frac{\sigma}{\nu}}\left(-\frac{\sigma}{\nu^{2}}\right)
$$

and differentiating again w.r.t. $\sigma$

$$
\psi_{\nu \sigma}=\frac{\partial \psi_{\nu}}{\partial \frac{\sigma}{\nu}} \frac{\partial \frac{\sigma}{\nu}}{\partial \sigma}=\frac{\partial \psi_{\nu}}{\partial \frac{\sigma}{\nu}}\left(\frac{1}{\nu}\right) .
$$

It follows that

$$
\psi_{\nu \nu}=\psi_{\nu \sigma}\left(-\frac{\sigma}{\nu}\right)
$$

which is a function of $\sigma / \nu$. Hence,

$$
\psi_{\nu \nu \nu}=\frac{\partial \psi_{\nu \nu}}{\partial \frac{\sigma}{\nu}} \frac{\partial \frac{\sigma}{\nu}}{\partial \nu}=\frac{\partial \psi_{\nu \nu}}{\partial \frac{\sigma}{\nu}} \frac{-\sigma}{\nu^{2}}
$$

and

$$
\psi_{\nu \nu \sigma}=\frac{\partial \psi_{\nu \nu}}{\partial \frac{\sigma}{\nu}} \frac{\partial \frac{\sigma}{\nu}}{\partial \sigma}=\frac{\partial \psi_{\nu \nu}}{\partial \frac{\sigma}{\nu}} \frac{1}{\nu}>0
$$

Positivity of $\psi_{\nu \nu \sigma}$ is shown in Franke et al (1998, Lemma 3), and hence

$$
\psi_{\nu \nu \nu}=\psi_{\nu \nu \sigma}\left(-\frac{\sigma}{\nu}\right)<0
$$

This proves the Lemma

\section{Proof of Claim 2:}

Rewrite equation (7) as

$$
A_{z, \varepsilon}(x)=\gamma \frac{1-\psi^{\prime}(x)}{x+z-\psi(x)} x
$$

Differentiating w.r.t. $x$ we have

$$
A_{z, \varepsilon}^{\prime}(x)=-\gamma \frac{\psi^{\prime \prime}(x) x}{x+z-\psi(x)}+\frac{A_{z, \varepsilon}(x)}{\gamma x}\left[\gamma-A_{z, \varepsilon}(x)\right] .
$$


Differentiating the first term on the right hand side of (9) w.r.t. $x$ yields

$$
-\gamma \frac{\psi^{\prime \prime \prime}(x) x}{x+z-\psi(x)}+\frac{\psi^{\prime \prime}(x)}{x+z-\psi(x)}\left[A_{z, \varepsilon}(x)-\gamma\right],
$$

which is positive, since $\psi^{\prime \prime \prime}(x)<0$, by Lemma 1 . Also, the second term in (9) clearly increases with $x$, since $A_{z, \varepsilon}^{\prime}(x)<0$. Hence, $A_{z, \varepsilon}^{\prime \prime}(x)>0$.

\section{Proof of Proposition 1:}

First, $\gamma \geq 1$ and $z \leq 0$ imply that $A_{z}(x) \geq \gamma \geq 1, A_{z}^{\prime}(x)<0$, and $A_{z}^{\prime \prime}(x)>0$. From Corollary 1(i) in Franke et al (2006), $A_{z, y}(x) \geq A_{z}(x)$. Also, since $u(x+z)$ is standard risk averse, $A_{z, \varepsilon}(x)>A_{z}(x)$. Second, from Claim 2, $A_{z, \varepsilon}(x)$ is declining and convex, and hence from Franke et al (2006, Corollary $1(\mathrm{i})), A_{z, \varepsilon, y}(x)>A_{z, \varepsilon}(x)$. This proves statement a) in Proposition 1.

Statement b) follows since, for $z \leq 0, A_{z}(x)$ is declining. Hence, by Claim 1 , $A_{z, \varepsilon}(x)$ is declining. From the appendix in Franke et al (2006) it then follows that $A_{z, y}(x)$ and $A_{z, \varepsilon, y}(x)$ are declining 
Table 1: Twelve Cases of Expected Non-Market Wealth, Additive Background Risk and Multiplicative Background Risk

\begin{tabular}{|c|c|c|c|c|c|c|c|}
\hline 1. & $\begin{array}{l}\text { Zero ENMW, } \\
\text { Non-Stochastic } \\
\text { No Rollover Risk } \\
z=0, \sigma_{\varepsilon}=0 \\
\sigma_{y}=0\end{array}$ & 2. & $\begin{array}{l}\text { Zero ENMW, } \\
\text { Non-Stochastic } \\
\text { Rollover Risk } \\
z=0, \sigma_{\varepsilon}=0 \\
\sigma_{y}=0.3\end{array}$ & 3. & $\begin{array}{l}\text { Zero ENMW, } \\
\text { Stochastic } \\
\text { No Rollover Risk } \\
z=0, \sigma_{\varepsilon}=30 \\
\sigma_{y}=0\end{array}$ & 4. & $\begin{array}{l}\text { Zero ENMW, } \\
\text { Stochastic } \\
\text { Rollover Risk } \\
z=0, \sigma_{\varepsilon}=30 \\
\sigma_{y}=0.3\end{array}$ \\
\hline 5. & $\begin{array}{l}\text { Negative ENMW, } \\
\text { Non-Stochastic } \\
\text { No Rollover Risk } \\
z=-20, \sigma_{\varepsilon}=0 \\
\sigma_{y}=0\end{array}$ & 6. & $\begin{array}{l}\text { Negative ENMW, } \\
\text { Non-Stochastic } \\
\text { Rollover Risk } \\
z=-20, \sigma_{\varepsilon}=0 \\
\sigma_{y}=0.3\end{array}$ & 7. & $\begin{array}{l}\text { Negative ENMW, } \\
\text { Stochastic } \\
\text { No Rollover Risk } \\
z=-20, \sigma_{\varepsilon}=30, \\
\sigma_{y}=0\end{array}$ & 8. & $\begin{array}{l}\text { Negative ENMW, } \\
\text { Stochastic } \\
\text { Rollover Risk } \\
z=-20, \sigma_{\varepsilon}=30 \\
\sigma_{y}=0.3\end{array}$ \\
\hline 9. & $\begin{array}{l}\text { Positive ENMW, } \\
\text { Non-Stochastic } \\
\text { No Rollover Risk } \\
z=30, \sigma_{\varepsilon}=0 \\
\sigma_{y}=0\end{array}$ & 10. & $\begin{array}{l}\text { Positive ENMW, } \\
\text { Non-Stochastic } \\
\text { Rollover Risk } \\
z=30, \sigma_{\varepsilon}=0 \\
\sigma_{y}=0.3\end{array}$ & 11. & $\begin{array}{l}\text { Positive ENMW, } \\
\text { Stochastic } \\
\text { No Rollover Risk } \\
z=30, \sigma_{\varepsilon}=30 \\
\sigma_{y}=0\end{array}$ & 12. & $\begin{array}{l}\text { Positive ENMW, } \\
\text { Stochastic } \\
\text { Rollover Risk } \\
z=30, \sigma_{\varepsilon}=30 \\
\sigma_{y}=0.3\end{array}$ \\
\hline
\end{tabular}

1. ENMW stands for the expected value of non-market wealth.

2. Rollover risk is the zero-mean multiplicative background risk which applies to total wealth.

3. $\sigma_{\varepsilon}$, is the standard deviation of the non-market wealth, at year 7

4. $\sigma_{y}$, is the standard deviation of the rollover risk, at year 7 


\section{Table 2: Portfolio Optimisation Example: Data}

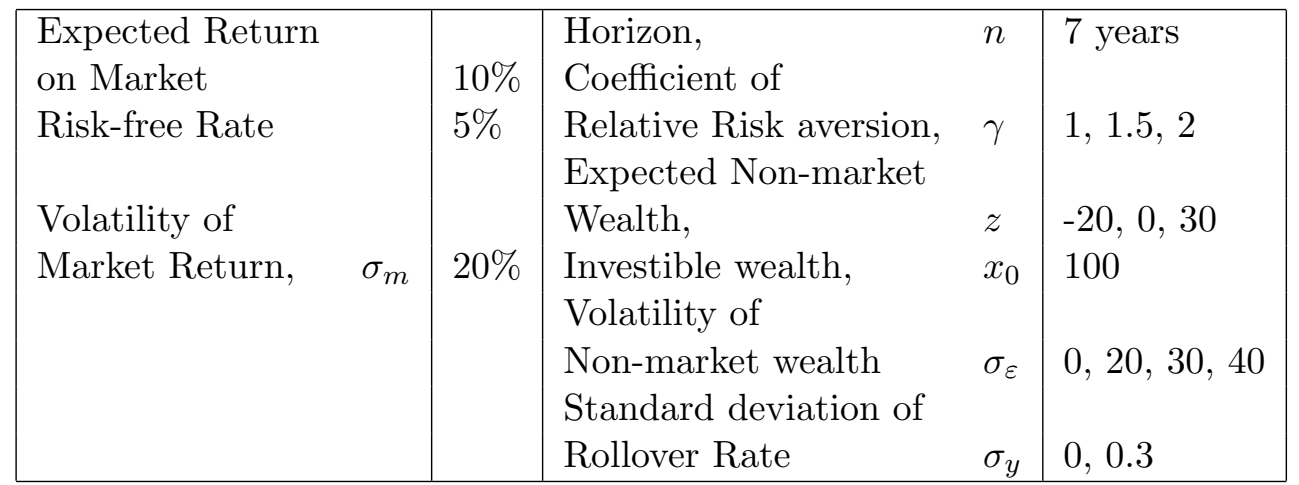

1. We assume that the market return follows a discrete binomial process, with a mean return of $10 \%$ over each year. The volatility of the underlying continuous process is $20 \%$.

2. The risk-free rate of interest is $5 \%$ on a discrete, annual basis.

3. In the right hand columns we show the investor characteristics. The horizon, when non-market wealth is realised is 7 years. The coefficient of relative risk aversion is $\gamma=1.5$. 


\section{Table 3: Multiplicative and Non-Market Wealth Risk Effects on Stock Proportions}

\begin{tabular}{|l|ccc|c|ccccccc|c|}
\hline \multicolumn{9}{|c|}{} & \multicolumn{9}{|c|}{ Year 6: state } & \\
\hline Case & $z$ & $\sigma_{\varepsilon}$ & $\sigma_{y}$ & Year 0 & 0 & 1 & 2 & 3 & 4 & 5 & 6 & Derived Utility \\
\hline $1(1.1,2.1)$ & 0 & 0 & 0 & 78 & 78 & 78 & 78 & 78 & 78 & 78 & 78 & CRRA \\
$2(2.2)$ & 0 & 0 & 0.3 & 78 & 78 & 78 & 78 & 78 & 78 & 78 & 78 & CRRA \\
$3(2.3)$ & 0 & 30 & 0 & 66 & 77 & 76 & 74 & 70 & 64 & 53 & 37 & DRRA \\
$4(2.4)$ & 0 & 30 & 0.3 & 55 & 76 & 74 & 71 & 64 & 51 & 31 & 15 & DRRA \\
\hline $5(1.2,3.1)$ & -20 & 0 & 0 & 67 & 74 & 72 & 70 & 68 & 65 & 61 & 57 & DRRA \\
$6(3.2)$ & -20 & 0 & 0.3 & 65 & 73 & 71 & 69 & 66 & 63 & 58 & 53 & DRRA \\
$7(3.3)$ & -20 & 30 & 0 & 53 & 72 & 69 & 65 & 58 & 49 & 35 & 22 & DRRA \\
$8(3.4)$ & -20 & 30 & 0.3 & 29 & 67 & 62 & 53 & 37 & 20 & 9 & 5 & DRRA \\
\hline $9(1.3,4.1)$ & 30 & 0 & 0 & 94 & 83 & 85 & 87 & 92 & 98 & 109 & 127 & IRRA \\
$10(4.2)$ & 30 & 0 & 0.3 & 96 & 83 & 86 & 89 & 93 & 100 & 111 & 131 & IRRA \\
$11(4.3)$ & 30 & 30 & 0 & 85 & 82 & 84 & 85 & 86 & 87 & 84 & 73 & $?$ (U-shape here) \\
$12(4.4)$ & 30 & 30 & 0.3 & 83 & 83 & 84 & 85 & 86 & 85 & 77 & 57 & $?($ U-shape here) \\
\hline
\end{tabular}

1. All data is as shown in Table 2. In column 1 the cases are numbered 1-12. In brackets we indicate the corresponding cases in Figures 1-4.

2. The state is indexed by the number of down-moves in the binomial process after 6 years.

3. $z$ is the expected non-market wealth of the investor at time $0 . \sigma_{y}$ is the standard deviation of the rollover rate. $\sigma_{\varepsilon}$ is the standard deviation of the non-market wealth process.

4. Column 3 shows the percentage of stocks in the optimal portfolio in year 0 .

5. Columns 4-8 show the percentage of stocks in the optimal portfolio in year 6 in the various states.

6. The derived utility is the utility for market wealth, $x$. It is of the type CRRA (constant relative risk aversion), DRRA (declining relative risk aversion), or IRRA (increasing relative risk aversion). RA stands for risk aversion.

7. In Cases 11 and 12, "?" indicates that, although in these examples the investor acts like an agent with increasing or drcreasing or U-Shaped relative 
risk aversion, this will not always be the case. Whether the agent acts as if DRRA or IRRA depends upon the size of $\sigma_{\varepsilon}$. 


\section{Table 4: Multiplicative and Non-Market Wealth Effects on Stock Proportions: Sensitivity Analysis}

\begin{tabular}{|l|cccc|c|ccccccc|c|}
\hline \multicolumn{10}{|c|}{} & \multicolumn{7}{|c|}{ Year 6: state } & \\
\hline Case & $\gamma$ & $z$ & $\sigma_{\varepsilon}$ & $\sigma_{y}$ & Year 0 & 0 & 1 & 2 & 3 & 4 & 5 & 6 & Derived Utility \\
\hline 11 & 1.5 & 30 & 30 & 0 & 85 & 82 & 84 & 85 & 86 & 87 & 84 & 73 & U-shape \\
$11 \mathrm{a}$ & 1.5 & 30 & 20 & 0 & 90 & 82 & 84 & 86 & 89 & 93 & 98 & 102 & IRRA \\
$11 \mathrm{~b}$ & 1.5 & 30 & 40 & 0 & 77 & 82 & 82 & 82 & 81 & 76 & 64 & 44 & DRRA \\
$11 \mathrm{c}$ & 2 & 30 & 20 & 0 & 63 & 63 & 62 & 63 & 63 & 63 & 61 & 57 & U-shape \\
$11 \mathrm{~d}$ & 1 & 30 & 30 & 0 & 129 & 122 & 124 & 128 & 132 & 134 & 121 & 69 & U-shape \\
\hline 12 & 1.5 & 30 & 30 & 0.3 & 83 & 83 & 84 & 85 & 86 & 85 & 77 & 57 & U-shape \\
$12 \mathrm{a}$ & 1.5 & 30 & 20 & 0.3 & 91 & 83 & 85 & 87 & 90 & 94 & 98 & 100 & IRRA \\
$12 \mathrm{~b}$ & 1.5 & 30 & 40 & 0.3 & 69 & 82 & 82 & 81 & 77 & 65 & 43 & 22 & DRRA \\
$12 \mathrm{c}$ & 2 & 30 & 30 & 0.3 & 61 & 63 & 64 & 63 & 63 & 61 & 57 & 49 & U-shape \\
$12 \mathrm{~d}$ & 1 & 30 & 30 & 0.3 & 125 & 122 & 125 & 128 & 132 & 131 & 99 & 32 & U-shape \\
\hline
\end{tabular}

1. All data is as shown in Table 2 .

2. The state is indexed by the number of down-moves in the binomial process after 6 years.

3. $z$ is the expected non-market wealth of the investor at time $0 . \sigma_{y}$ is the (nonannualised) standard deviation of the rollover rate. $\sigma_{\varepsilon}$ is the (annualised) volatility of the non-market wealth process.

4. Column 3 shows the percentage of stocks in the optimal portfolio in year 0 .

5. Columns 4-8 show the percentage of stocks in the optimal portfolio in year 6 in the various states.

6. The derived utility is the utility for market wealth, $x$. It is of the type CRRA (constant relative risk aversion), DRRA (declining relative risk aversion), or IRRA (increasing relative risk aversion). RA stands for risk aversion.

7. In Cases 11 and 12, "?" indicates that, although in these examples the investor acts like an agent with increasing or drcreasing or U-Shaped relative risk aversion, this will not always be the case. Whether the agent acts as if DRRA or IRRA depends upon the size of $\sigma_{\varepsilon}$. 
1. Aït-Sahalia, Y. and A.W. Lo, (2000), "Nonparametric Risk Management and Implied Risk Aversion," Journal of Econometrics 94, 9-51.Bodie, Z. (2003), "Thoughts on the Future: Life-Cycle Investing in Theory and Practice", Financial Analysts Journal, Jan/Febr, 24 - 29.

2. Back, K. and P. H. Dybvig, (1993), "On Existence of Optimal Portfolios in Complete Markets," Washington University in St. Louis, Working Paper.

3. Bodie, Z., Merton, R. and Samuelson, W.F. (1992) "Labor Supply Flexibility and Portfolio Choice in a Life Cycle Model," Journal of Economic Dynamics and Control, 16, 427-449.

4. Brennan, M.J., Schwartz, E. and Lagnado, R. (1997), "Strategic Asset Allocation," Journal of Economic Dynamics and Control, 21, 1377-1403.

5. Brennan, M.J. and Xia, Y. (2002), "Dynamic Asset Allocation under Inflation," Journal of Finance, 57, 1201-1238.

6. Campbell, J. and Viceira, L.M. (2002), Strategic Asset Allocation - Portfolio Choice for Long-Term Investors, Oxford Univ. Press.

7. Cocco, J.F. (2005), "Portfolio Choice in the Presence of Housing," Review of Financial Studies, 18, 2, 535-567.

8. Cocco, J.F., Gomes, F.J. and P.J. Maenhout (2005), "Consumption and Portfolio Choice over the Life Cycle," Review of Financial Studies, 18, 2, 495-533.

9. Eeckhoudt, L. and Kimball, M. (1992), "Background Risk, Prudence and the Demand for Insurance," in G. Dionne (ed.), Contributions to Insurance Economics, Kluwer Academic Publishers, 239-254.

10. Franke, G, Schlesinger, H and Stapleton, R C (2006), "Multiplicative Background Risk", Management Science, 52, 146-153.

11. Franke, G., Stapleton, R.C. and Subrahmanyam, M.G. (1998), "Who Buys and Who Sells Options: The Role of Options in an Economy with Background Risk," Journal of Economic Theory, 82, 89-109.

12. Gollier, C. (2001), The Economics of Risk and Time, MIT Press. 
13. Gollier, C. and Kimball, M. (1996), "Towards a Systematic Approach to the Economics of Uncertainty: Characterizing Utility Functions," Unpublished Working Paper, University of Michigan.

14. Gollier, C. and Pratt, J. (1996), "Risk Vulnerability and the Tempering Effect of Background Risk," Econometrica 64, 1109-1124.

15. Heaton, J. and Lucas, D. (1997), "Market Frictions, Savings Behavior and Portfolio Choice," Macroeconomic Dynamics, 1, 76-101.

16. Ingersoll, J. (1987), Theory of Financial Decision Making, Rowman and Littlefield.

17. Jackwerth, J.C., (2000), "Recovering Risk Aversion from Option Prices and Realized Returns," Review of Financial Studies 13, 433-451.

18. Kim, T.S. and Omberg, E. (1996), "Dynamic Nonmyopic Portfolio Behavior," Review of Financial Studies, 9, 141-161.

19. Koo, H.K. (1998), "Consumption and Portfolio Selection with Labor Income: A Continuous Time approach," Mathematical Finance, 8, 49-65.

20. Merton, R. (1969), "Lifetime Portfolio selection Under Uncertainty," Review of Economics and Statistics, 51, 247-257.

21. Merton, R. (1971), "Optimal Consumption and Portfolio Rules in a ContinuousTime Model," Journal of Economic Theory 3, 373-413.

22. Merton, R.C. (2003), "Thoughts on the Future: Theory and Practice in Investment Management," Financial Analysts Journal, Jan/Feb, 17-23.

23. Meyer, D.J. and Meyer, J. (2005), "Relative Risk Aversion: What Do We Know?" Journal of Risk and Uncertainty, 31, 243-262.

24. Rothschild, M. and Stiglitz, J. (1970), "Increases in Risk I: A Definition," Journal of Economic Theory 2, 225-243.

25. Shiller, R. (2003), The New Financial Order: Risk in the 21st Century, Princeton University Press.

26. Viceira, L.M. (2001) "Optimal Portfolio Choice for Long-horizon Investors with Nontradable Labor Income," Journal of Finance, 56, 433-470. 
Notes for Figures

In Figures 1-5, the trio $\left(z, \sigma_{\varepsilon}, \sigma_{y}\right)$ signifies the levels of expected non-market wealth, the risk of non-market wealth and the $\widetilde{y}$ risk.

1. Figure $8 \mathrm{~b})$ depicts the observed RRA $A_{z}(x)$ for $\gamma=1.5$ and positive fixed non-market wealth $z=30$ and the observed RRA $A_{z, \varepsilon}(x)$ for risky nonmarket wealth with expectation 30 . The probability distribution of $\varepsilon$ is : $\varepsilon= \pm 60$ with probability $1 / 16, \varepsilon= \pm 30$ with probability $4 / 16$, and $\varepsilon=0$ with probability $6 / 16$.

2. In Figure 1, we plot the logarithm of the total portfolio gross return over the 7 years against the logarithm of the market gross return, for the case where there is no non-market wealth risk and no $\widetilde{y}$ risk. The expected return on the market is $10 \%$ and the volatility of the market is $20 \%$. The coefficient of relative risk aversion is $\gamma=1.5$. The expected non-market wealth is $z=0$ in case $1.1, z=30$ in case 1.2 , and $z=-20$ in case 1.3 .

3. In Figure 2, we compare four different scenarios. In each case, the expected value of the non-market wealth is $z=0$, while the market data and the cofficient of relative risk aversion is the same as in the example in Figure 1. In case 2.1 we assume that there is no risk, i.e. neither non-market wealth risk nor $\widetilde{y}$ risk. In case 2.2, the risk of the non-market wealth is $\sigma_{\varepsilon}=0$ and the $\widetilde{y}$ risk $\sigma_{y}=0.3$. In case 2.3 , the risk of the non-market wealth is $\sigma_{\varepsilon}=30$ and the $\widetilde{y}$ risk $\sigma_{y}=0$. In case 2.4, the risk of the non-market wealth is $\sigma_{\varepsilon}=30$ and the $\widetilde{y}$ risk is $\sigma_{y}=0.3$.

4. In Figure 3, we compare four different scenarios. In each case, the expected value of the non-market wealth is $z=-20$, while the market data and the cofficient of relative risk aversion is the same as in the example in Figure 1. In case 3.1 we assume that there is no risk, i.e. neither non-market wealth risk nor $\widetilde{y}$ risk. In case 3.2, the risk of the non-market wealth is $\sigma_{\varepsilon}=0$ and the $\widetilde{y}$ risk $\sigma_{y}=0.3$. In case 3.3, the risk of the non-market wealth is $\sigma_{\varepsilon}=30$ and the $\widetilde{y}$ risk $\sigma_{y}=0$. In case 3.4, the risk of the non-market wealth is $\sigma_{\varepsilon}=30$ and the $\widetilde{y}$ risk is $\sigma_{y}=0.3$.

5. In Figure 4, we compare four different scenarios. In each case, the expected value of the non-market wealth is $z=30$, while the market data and the 
cofficient of relative risk aversion is the same as in the example in Figure 1. In case 4.1 we assume that there is no risk, i.e. neither non-market wealth risk nor $\widetilde{y}$ risk. In case 4.2 , the risk of the non-market wealth is $\sigma_{\varepsilon}=0$ and the $\widetilde{y}$ risk $\sigma_{y}=0.3$. In case 4.3 , the risk of the non-market wealth is $\sigma_{\varepsilon}=30$ and the $\widetilde{y}$ risk $\sigma_{y}=0$. In case 4.4, the risk of the non-market wealth is $\sigma_{\varepsilon}=30$ and the $\widetilde{y}$ risk is $\sigma_{y}=0.3$.

6. In Figure 5, we illustrate the dynamic asset allocation strategy in the case where expected non-market wealth is $z=30, \sigma_{\varepsilon}=0$ and the $\widetilde{y}$ risk is $\sigma_{y}=0.3$. This is the same as case 8 in Table 3 . The line marked $0 d$ shows the stock proportion in the state where there have been no down moves in the market return process by year $t$. The line marked $1 d$ shows the stock proportion in the state where there has been one down move in the market return process by year $t$, and so on.

7. In Figure 6, we illustrate the dynamic asset allocation strategy in the case where expected non-market wealth is $z=0, \sigma_{\varepsilon}=30$ and the $\widetilde{y}$ risk is $\sigma_{y}=0.3$. This is the same as case 4 in Table 3. The line marked $0 d$ shows the stock proportion in the state where there have been no down moves in the market return process by year $t$. The line marked $1 d$ shows the stock proportion in the state where there has been one down move in the market return process by year $t$, and so on.

8. In Figure 7, we illustrate the dynamic asset allocation strategy in the case where expected non-market wealth is $z=30, \sigma_{\varepsilon}=30$ and the $\widetilde{y}$ risk is $\sigma_{y}=0$. This is the same as case 11 in Table 3 . The line marked $0 d$ shows the stock proportion in the state where there have been no down moves in the market return process by year $t$. The line marked $1 d$ shows the stock proportion in the state where there has been one down move in the market return process by year $t$, and so on.

9. In Figure 8, we illustrate the dynamic asset allocation strategy in the case where expected non-market wealth is $z=0, \sigma_{\varepsilon}=30$ and the $\widetilde{y}$ risk is $\sigma_{y}=0.3$. This is the same as case 12 in Table 3 . The line marked $0 d$ shows the stock proportion in the state where there have been no down moves in the market return process by year $t$. The line marked $1 d$ shows the stock proportion in the state where there has been one down move in the market return process by year $t$, and so on. 


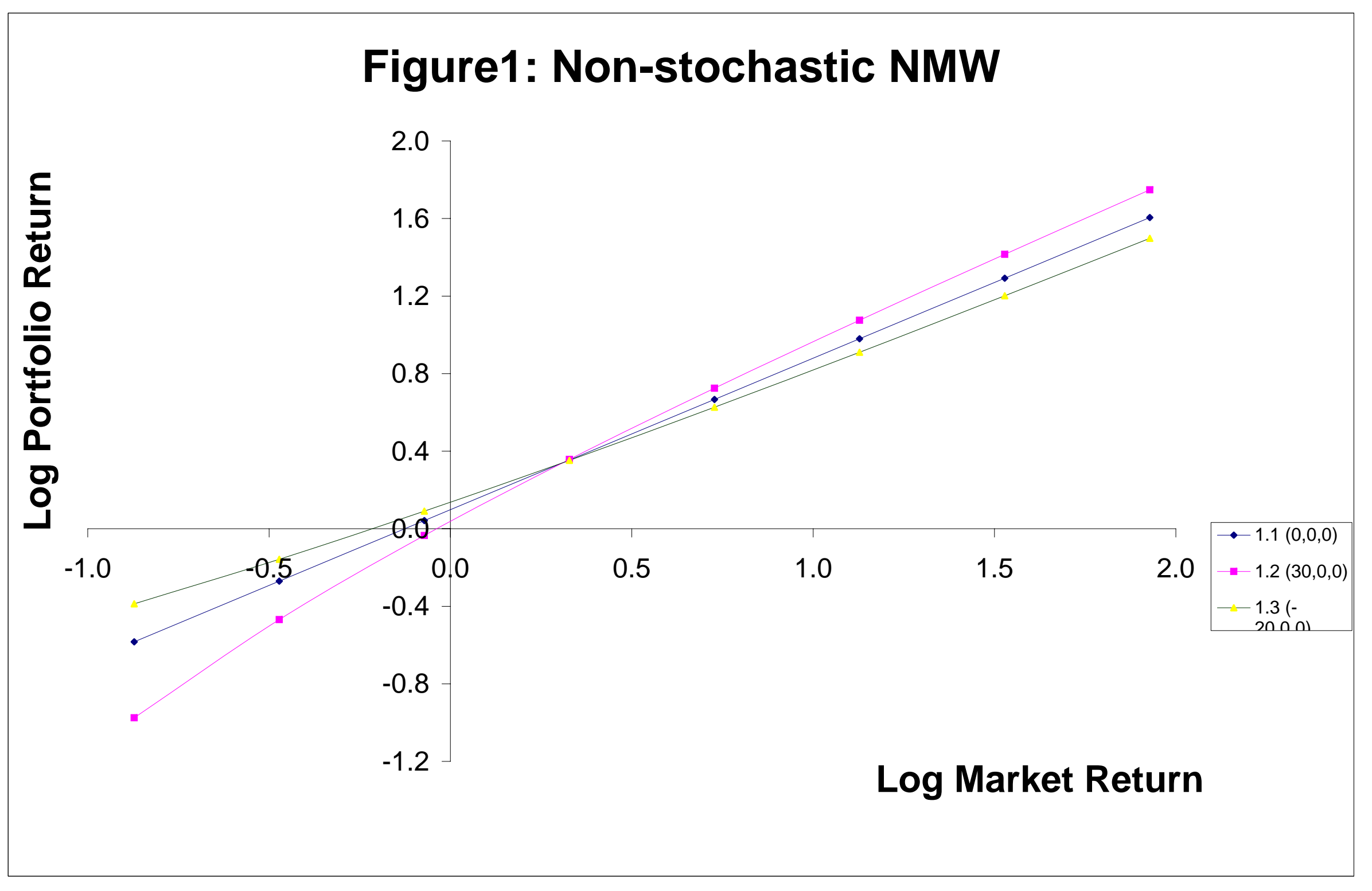




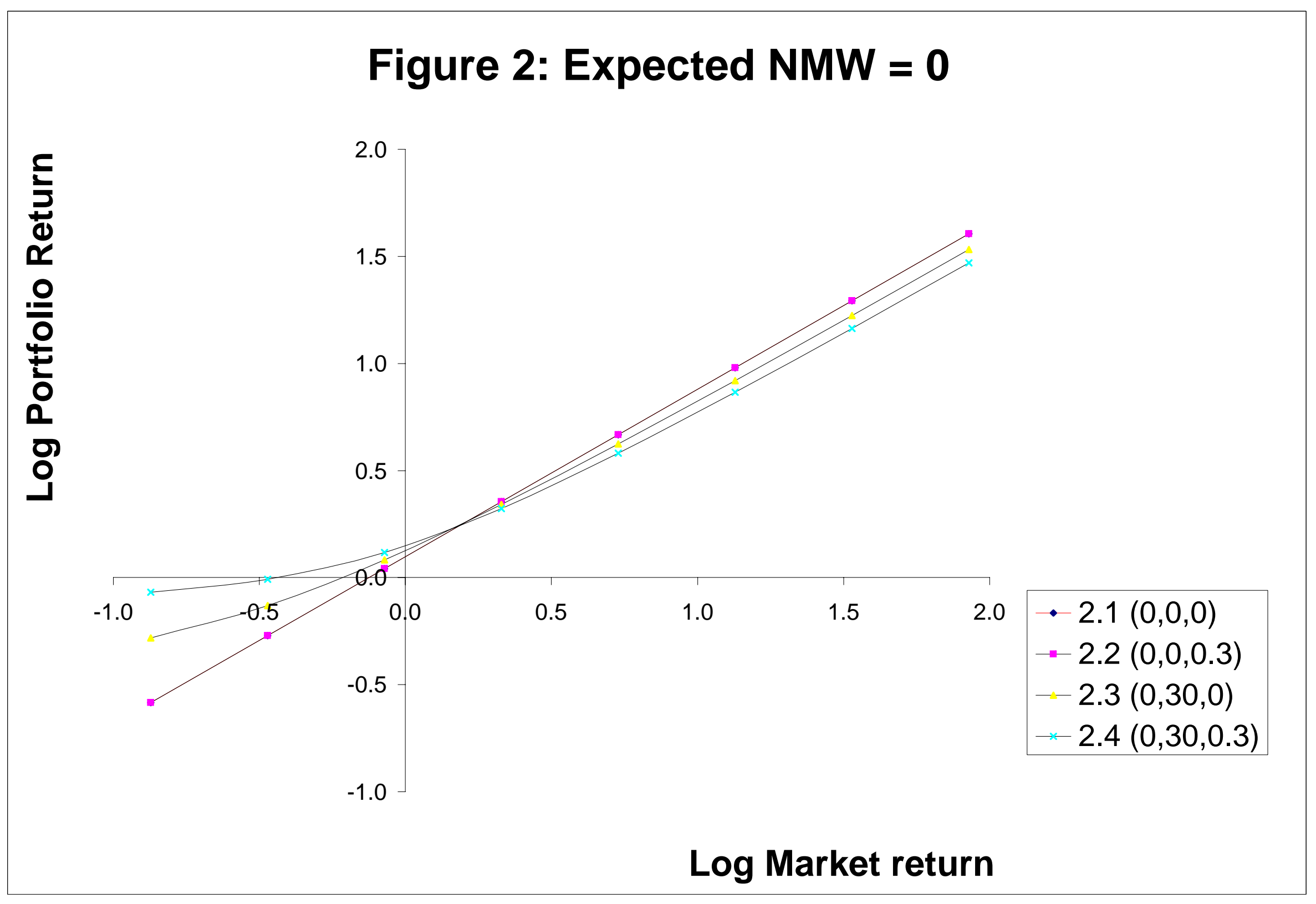




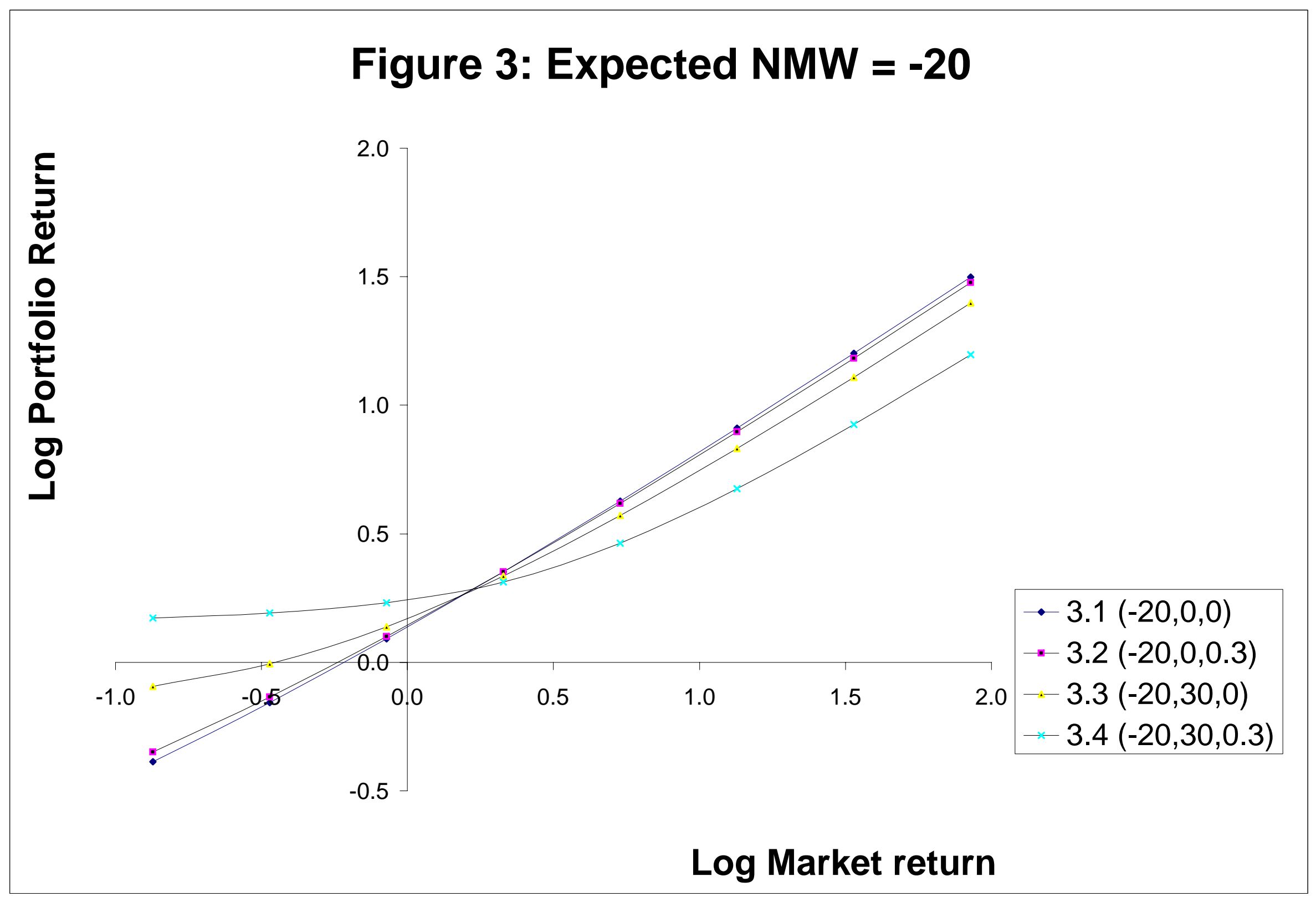




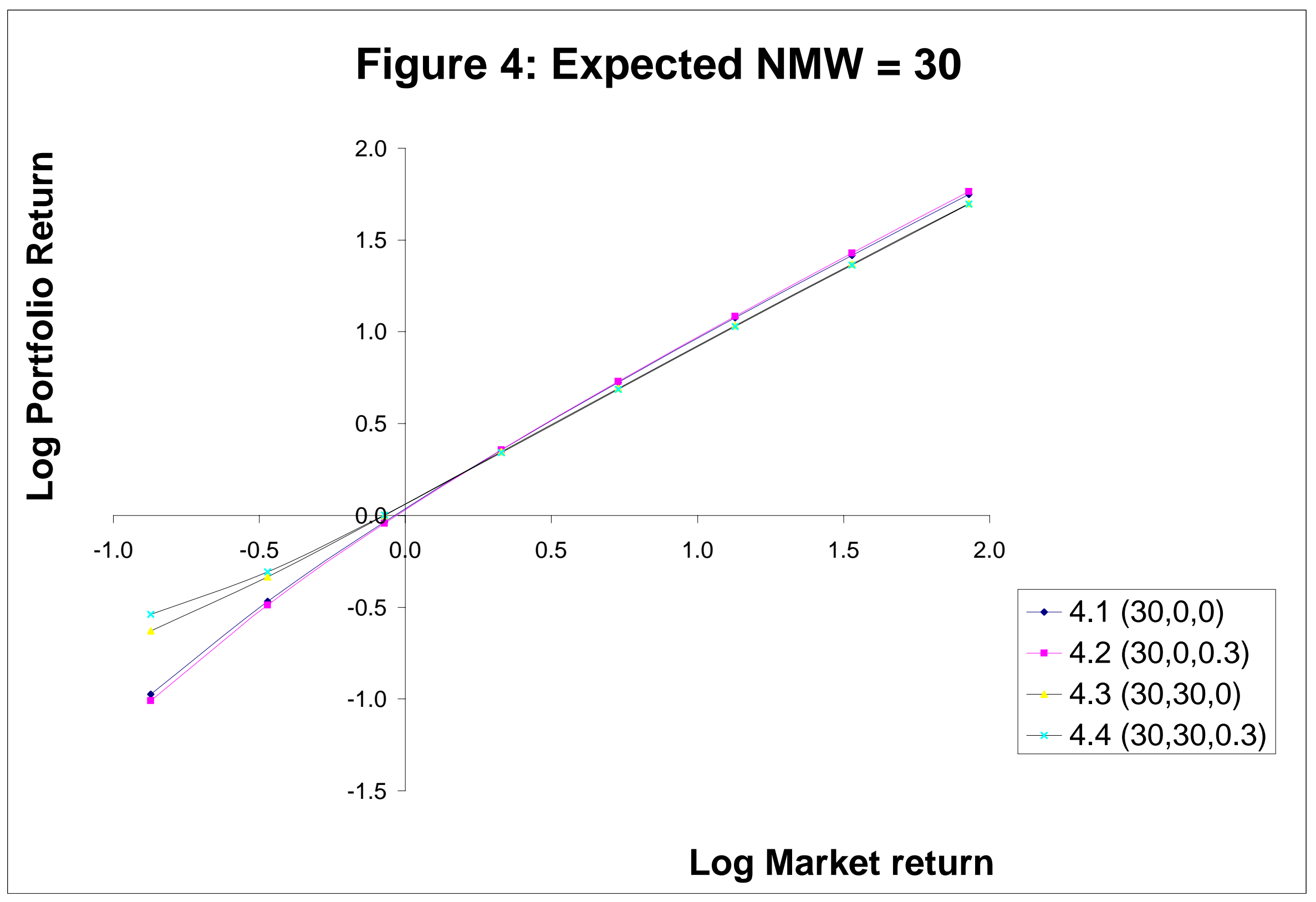




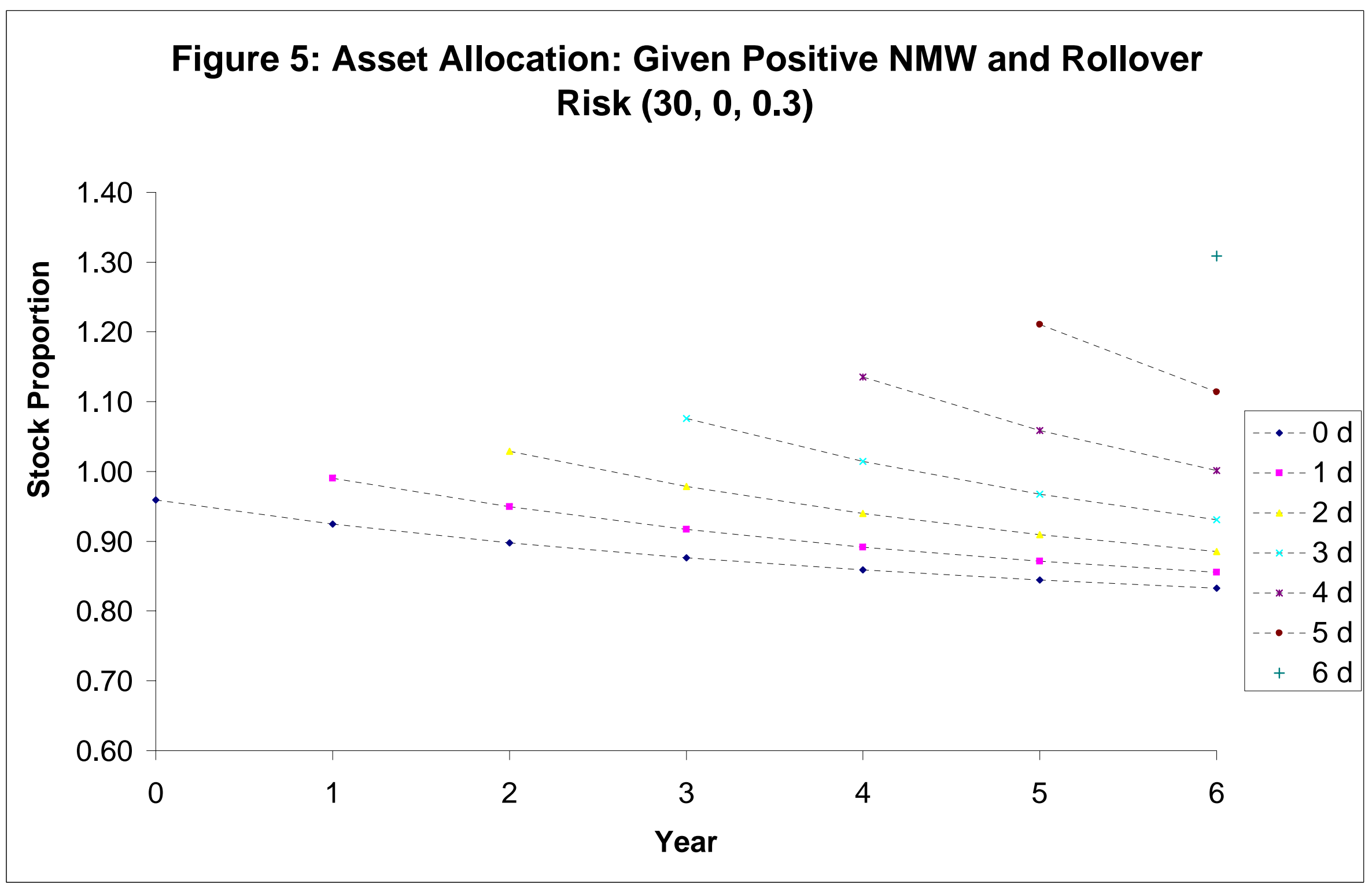




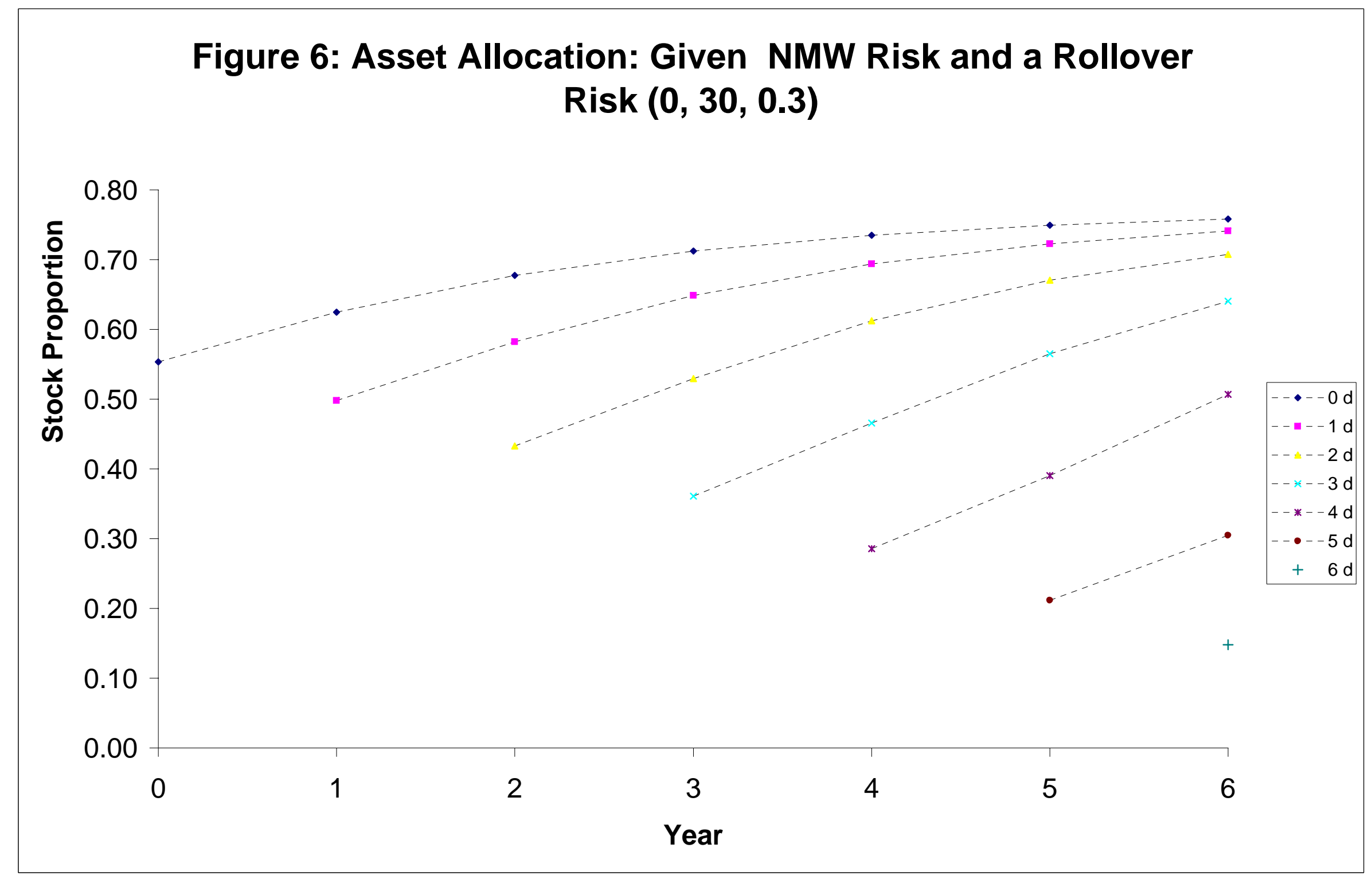




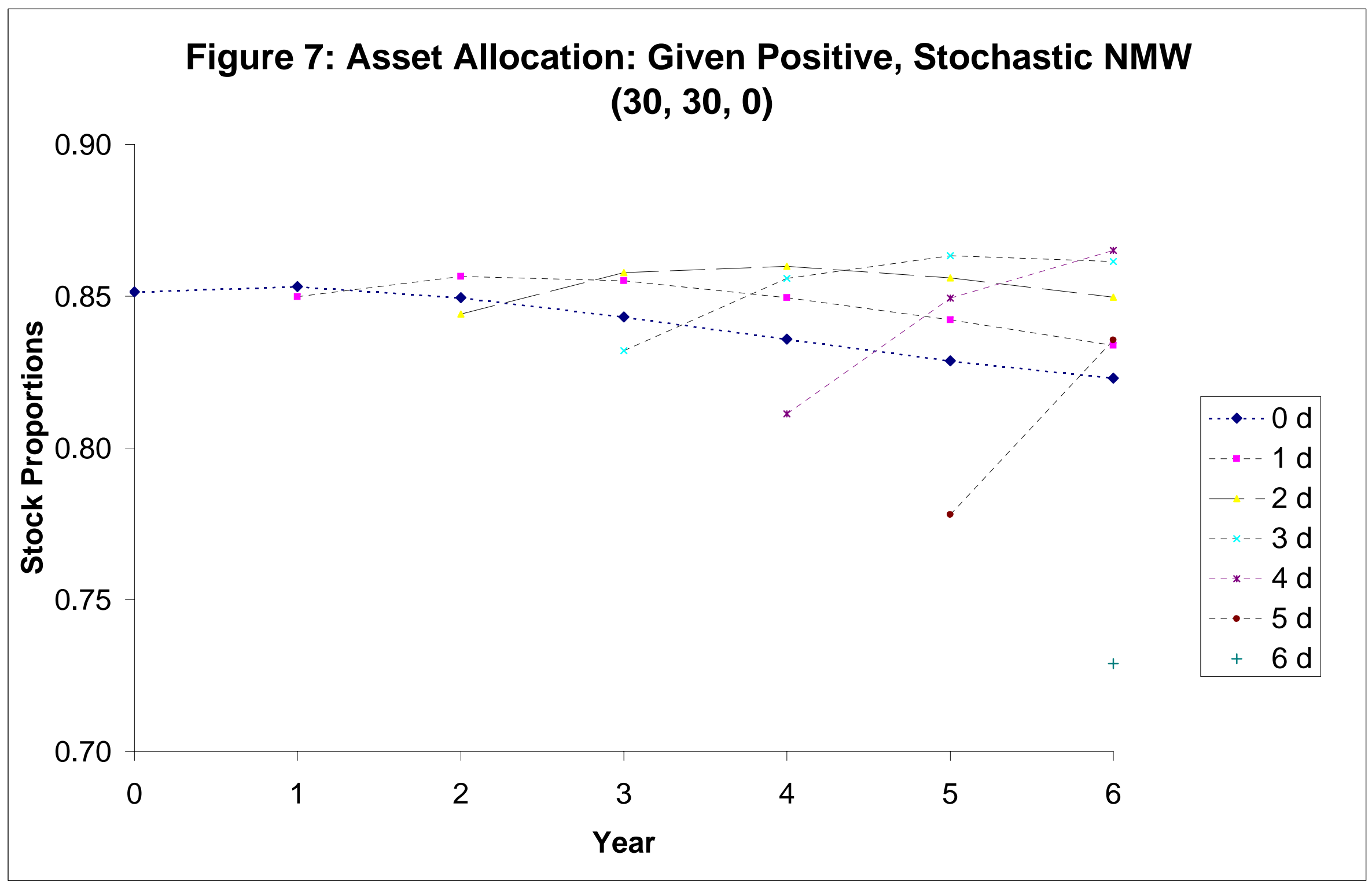




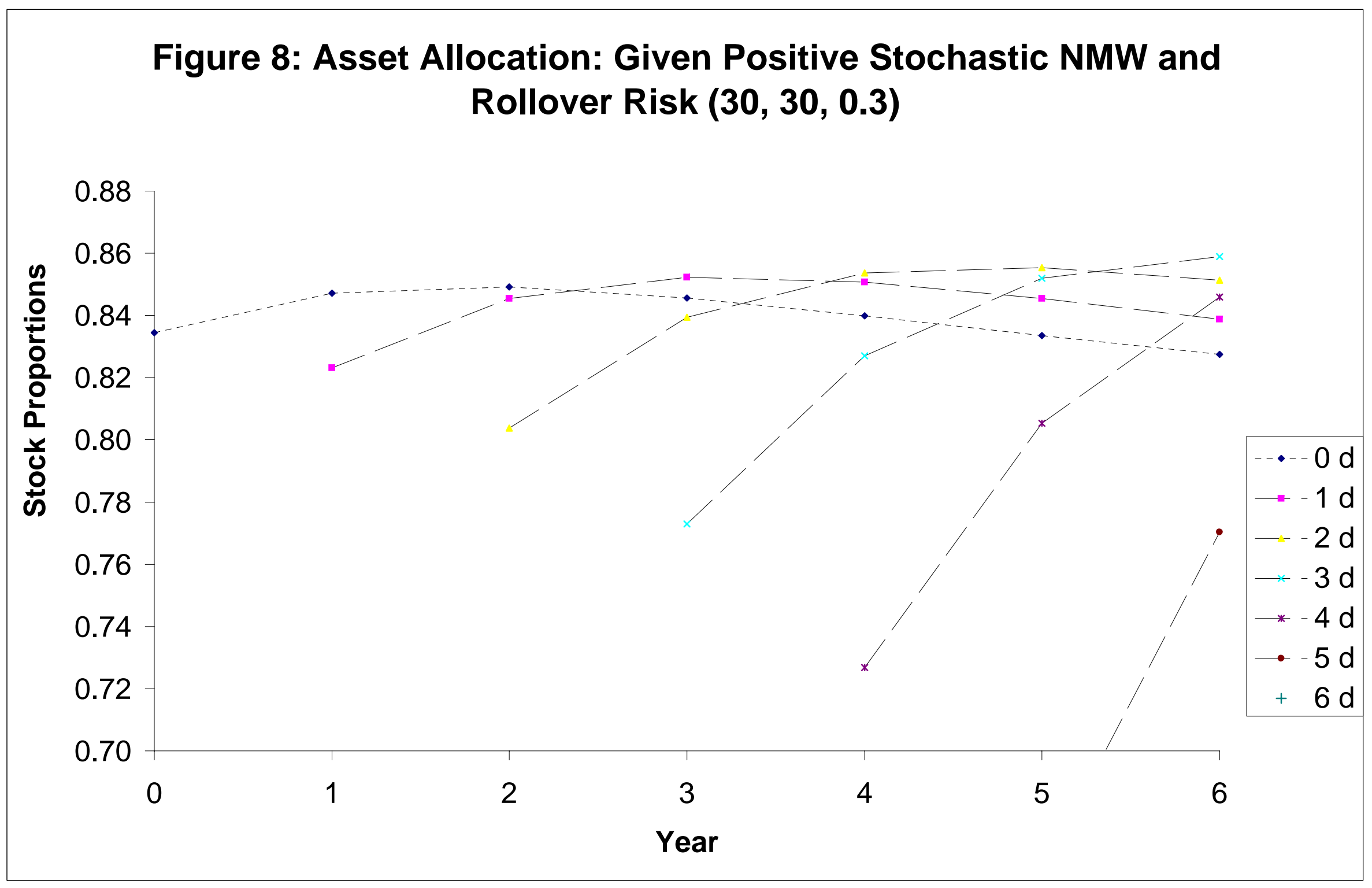


Figure 8a): Derived Relative Risk Aversion

$\gamma=3 ; z=30 ; A_{z}(x)$ is the derived relative risk aversion for $\varepsilon \equiv 0, A_{z, \varepsilon}(x)$ the derived relative risk aversion for $\varepsilon$ being symmetrically distributed around zero with $\operatorname{Prob}(-29)=\operatorname{Prob}(+29)=0.0625, \operatorname{Prob}(-10)=\operatorname{Prob}(+10)=0.25$ and $\operatorname{Prob}(0)=0.375$.
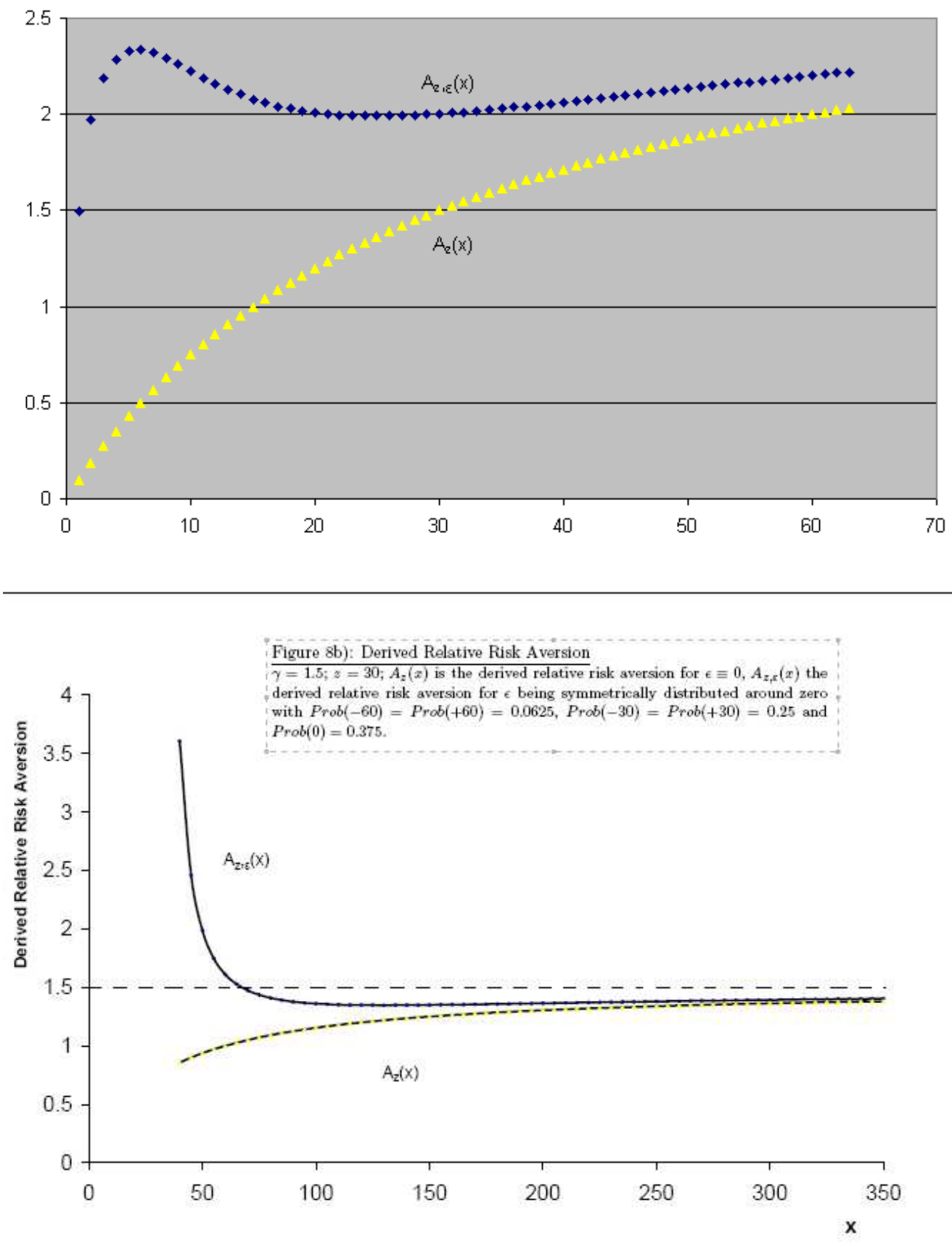